\title{
Pulsatile flow and mass transport past a circular cylinder
}

\author{
Jennifer R. Zierenberg, Hideki Fujioka, and Vinod Suresh \\ Department of Biomedical Engineering, University of Michigan, Ann Arbor, Michigan 48109-2099 \\ Robert H. Bartlett and Ronald B. Hirschl \\ Department of Surgery, University of Michigan Medical Center, Ann Arbor, Michigan 48109 \\ James B. Grotberg ${ }^{\text {a) }}$ \\ Department of Biomedical Engineering, University of Michigan, Ann Arbor, Michigan 48109-2099
}

(Received 15 July 2005; accepted 13 December 2005; published online 27 January 2006)

\begin{abstract}
The mass transport of a pulsatile free-stream flow past a single circular cylinder is investigated as a building block for an artificial lung device. The free stream far from the cylinder is represented by a time-periodic (sinusoidal) component superimposed on a steady velocity. The dimensionless parameters of interest are the steady Reynolds number $(\mathrm{Re})$, Womersley parameter $(\alpha)$, sinusoidal amplitude $(A)$, and the Schmidt number $(\mathrm{Sc})$. The ranges investigated in this study are $5 \leqslant \operatorname{Re}$ $\leqslant 40,0.25 \leqslant \alpha \leqslant 4,0.25 \leqslant A \leqslant 0.75$, and $\mathrm{Sc}=1000$. A pair of vortices downstream of the cylinder is observed in almost all cases investigated. These vortices oscillate in size and strength as $\alpha$ and $A$ are varied. For $\alpha<\alpha_{c}$, where $\alpha_{c}=0.005 A^{-1.13} \mathrm{Re}^{1.33}$, the vortex is always attached to the cylinder (persistent); while for $\alpha>\alpha_{c}$, the vortex is attached to the cylinder only during part of a time cycle (intermittent). The time-averaged Sherwood number, $\overline{\overline{\mathrm{Sh}}}$, is found to be largely influenced by the steady Reynolds number, increasing approximately as $\operatorname{Re}^{1 / 2}$. For $\alpha=0.25, \overline{\overline{S h}}$ is less than the steady $(\alpha=0, A=0)$ value and decreases with increasing $A$. For $\alpha=2$ and $\alpha=4, \overline{\overline{S h}}$ is greater than the steady value and increases with increasing $A$. These qualitatively opposite effects of pulsatility are discussed in terms of quasisteady versus unsteady transport. The maximum increase over steady transport due to pulsatility varies between $14.4 \%$ and $20.9 \%$ for $\operatorname{Re}=10-40, \alpha=4$, and $A=0.75$.
\end{abstract}

(C) 2006 American Institute of Physics. [DOI: 10.1063/1.2164475]

\section{INTRODUCTION}

An artificial lung is currently being developed as a bridge to lung transplantation for patients suffering from chronic lung disease. ${ }^{1-4}$ This device is perfused by the pulsating blood flow leaving the right heart and is designed to provide complete gas exchange support. It consists of a chamber containing hollow, microporous fibers through which air flows. Deoxygenated blood enters the center of the device and flows radially across the fiber bundle, where oxygen from inside the fibers is exchanged with carbon dioxide from the blood flowing over them. The oxygenated blood then exits the device.

As a fundamental study for the artificial lung, the fluid dynamics and mass transfer associated with pulsatile flow past a single fiber or cylinder can be studied for the operating parameter range of the artificial lung, namely small Reynolds number $(\mathrm{Re})$ and large Schmidt number $(\mathrm{Sc})$, where $\mathrm{Re}$ $=2 a U_{o} \rho / \mu$ and $\mathrm{Sc}=\mu / \rho D$, with cylinder radius $a$, freestream velocity $U_{o}$, fluid density $\rho$, fluid viscosity $\mu$, and molecular diffusivity $D$. Other engineering applications for pulsatile flow past a cylinder with its associated mass/heat transport include hot-wire anemometry, heat exchangers, and corrosion of horizontal cylinders, among others. Most of the relevant heat transfer measurements of the Nusselt number

\footnotetext{
${ }^{a)}$ Author to whom correspondence should be addressed. Telephone: (734) 936-3834. Fax: (734) 936-1905. Electronic mail: grotberg@umich.edu
}

have been done in air and water, i.e., at a low Prandtl number $(0.7 \leqslant \operatorname{Pr} \leqslant 6)$, where $\operatorname{Pr}$ is analogous to Sc with the molecular diffusivity replaced by the thermal diffusivity. Few data are available for mass/heat transfer in the high Schmidt/ Prandtl number range that would correspond to our application.

The available data for high Schmidt number concern steady flow past a single cylinder. Many correlations for the mean Sherwood/Nusselt number have been developed for steady flow past a cylinder based on experimental measurements and can be applied for a large range of Schmidt numbers, including high Schmidt numbers. One of the classic and simplest correlations was developed by Hilpert. ${ }^{5}$ It expresses the Nusselt number as $\mathrm{Nu}=C \operatorname{Re}^{n} \operatorname{Pr}^{1 / 3}$, where the constants $C$ and $n$ are specified for different Reynolds number ranges and are valid for $\operatorname{Re}=0.4-4 \times 10^{5}$ and $\operatorname{Pr} \geqslant 0.7$; for $4 \leqslant \operatorname{Re}$ $\leqslant 40, C=0.911$ and $n=0.385$. Vogtlander and Bakker ${ }^{6}$ experimentally studied mass transfer from a liquid to a wire for $5 \leqslant \operatorname{Re} \leqslant 400$ and $1300 \leqslant \mathrm{Sc} \leqslant 2000$. They showed good agreement between their data and a correlation by Van der Hegge Zijnen, ${ }^{7} \mathrm{Sh}=0.38 \mathrm{Sc}^{0.2}+\left(0.56 \mathrm{Re}^{0.5}+0.001 \mathrm{Re}\right) \mathrm{Sc}^{0.33}$, which is valid for $1000<\mathrm{Sc}<2000$. For $1 \leqslant \mathrm{Re} \leqslant 100$, it is found that $\mathrm{Sh} \sim \operatorname{Re}^{1 / 2} \mathrm{Sc}^{1 / 3}$ in Ref. 6. Gnielinski ${ }^{8}$ proposed a correlation for forced convection flow past a cylinder in the laminar regime, $\mathrm{Nu}=0.664 \mathrm{Re}^{1 / 2} \mathrm{Pr}^{1 / 3}$, valid for $10<\mathrm{Re}$ $<10^{7}$ and $0.6<\operatorname{Pr}<1000$. Kurdyumov and Fernandez ${ }^{9}$ developed a correlation formula for Nusselt number based on an asymptotic expansion for $\operatorname{Pr} \rightarrow \infty$ for steady flow past a 
cylinder. This analysis is only valid for small Reynolds numbers Re $\leq 5$. Recently, Sparrow ${ }^{10}$ considered the archival correlations for noncircular and circular cylinders and spheres in cross flow. He provides a new correlation for a cylinder that better represents gases for $O(1)$ Schmidt numbers and liquids for higher Schmidt numbers: $\mathrm{Nu}=0.25+\left(0.4 \mathrm{Re}^{1 / 2}\right.$ $\left.+0.06 \operatorname{Re}^{2 / 3}\right) \operatorname{Pr}^{0.37}\left(\mu / \mu_{\text {wall }}\right)^{1 / 4}$, where $\mu$ is the viscosity of the free-stream fluid and $\mu_{\text {wall }}$ is the viscosity of the fluid at the cylinder surface. It is valid for $1 \leqslant \operatorname{Re} \leqslant 10^{5}$.

One of the earliest studies of the effect of oscillations on heat transfer was performed by Van der Hegge Zijnen. ${ }^{7} \mathrm{He}$ experimentally studied cross flow of air past a thin wire in a flow with steady and fluctuating components. He observed a decrease in $\mathrm{Nu}$ due to the fluctuations compared to the steady $\mathrm{Nu}$ for small Reynolds numbers (Re $\leqslant 5$ ) with a maximum decrease of $4.3 \%$ for the highest fluctuation amplitude of $45 \%$ of the free-stream velocity. However, the frequency of the oscillation is not provided by the author. Leung et al. ${ }^{11}$ experimentally studied air cross flow past a cylinder oscillating in-line with the flow for $\mathrm{Re}=3000-50000$ for two oscillation amplitudes, 0.5 and $0.25 \mathrm{~mm}$, and two frequencies, 10 and $30 \mathrm{~Hz}$, relative to those for stationary cylinders. They observed that heat transfer is enhanced for both frequencies for $\operatorname{Re}<15000$, with further enhancement for larger oscillation amplitudes. However, at large Reynolds numbers (Re $>25000$ ), oscillations resulted in a decrease in the heat transfer rate. Karanth et al. ${ }^{12}$ numerically studied heat transfer for flow past an oscillating cylinder for $\mathrm{Re}=200$ and $\mathrm{Pr}$ $=1$ for velocity amplitudes $\left(A_{x}\right)$ of 0.25 and 0.5 relative to the free-stream velocity and a Strouhal number of 0.2 defined as $\mathrm{St}=\omega a / U_{o}$, where $\omega$ is the angular frequency of oscillation. They reported an increase in the mean Nusselt number due to cylinder oscillations and found the mean Nusselt number to increase with oscillation amplitude, $5.6 \%$ for $A_{x}=0.25$ and $5.8 \%$ for $A_{x}=0.5$ relative to the steady state. In their work, asymmetrical vortex shedding was induced by slightly rotating the cylinder counterclockwise and then clockwise with a constant angular velocity. The asymmetry of the vortex shedding had little effect on the isotherm fields near the cylinder, which are shown to be nearly symmetric.

The effect of a pulsating free-stream air flow past a stationary cylinder on heat transfer has been experimentally studied by Perwaiz and Base ${ }^{13}$ for $\mathrm{Re}=2000-14$ 000, amplitude of oscillation of $35 \%$ relative to the free-stream velocity, and a Strouhal number, based on the mean free-stream velocity, of $\sim 0.01-0.15$. They found a decrease in heat transfer compared to the steady state for the lower pulsation frequencies in their range, and the opposite for the higher pulsation frequencies. Sung et al. ${ }^{14}$ experimentally studied mass transfer from a sublimating naphthalene cylinder in pulsatile flow for $4500 \leqslant \operatorname{Re} \leqslant 12450 \quad(\mathrm{Sc} \approx 2.5)$, amplitude of pulsation $\sim 0.08-0.23$, and frequency $\sim 10-40 \mathrm{~Hz}$. Their measurements in the downstream separation region indicated an augmentation in mass transfer due to flow pulsation, with greater augmentation found for higher frequencies. Local mass transfer also increased with pulsation amplitude. In addition, it was found that at large Reynolds numbers the effect of pulsation on mass transfer decreases. Badr ${ }^{15}$ numerically studied the effect of free-stream fluctuations on the time-
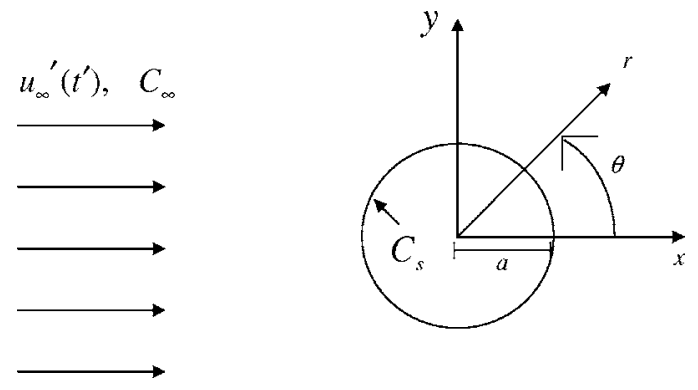

FIG. 1. Schematic of unsteady flow over and transport from a single cylinder.

averaged Nusselt number for $\mathrm{Re}=50-500(\mathrm{Pr}=0.7)$, amplitude of pulsation of $20 \%$ and $50 \%$ of the free-stream velocity, and $\mathrm{St}=\pi / 4-\pi$. He found that the Nusselt number increased with increasing amplitude and decreased with increasing frequency. In addition, a pulsating free stream had a greater effect on Nusselt number for higher Reynolds numbers. In his analysis, symmetry about the center line of the cylinder was imposed as part of the flow and thermal field solution.

Unsteady heat transfer from a cylinder with constant temperature in cross flow was numerically studied by Karniadakis ${ }^{16}$ for $\operatorname{Re} \leqslant 200$ and $\operatorname{Pr}=0.7$. Both constant heat flux at the cylinder surface and constant cylinder surface temperature cases were studied. Goldstein et al. ${ }^{17}$ experimentally investigated the effect of a wall boundary layer on mass transfer from a sublimating naphthalene cylinder ( $\mathrm{Sc}$ $=2.5$ ) in cross flow for $\mathrm{Re}=19000$. In particular, the effect of three-dimensional secondary flows in the form of horseshoe vortices near the wall-attached ends of the cylinder is discussed. In these areas, increases of $90 \%$ to $700 \%$ as compared to two-dimensional flow mass transfer are found at the front of the cylinder. Tiwari et al. ${ }^{18}$ numerically studied heat transfer in a rectangular channel with a built-in cylinder for $\operatorname{Re} \sim 3000-7000$ and $\operatorname{Pr}=0.7$. In this case, both the walls and cylinder were maintained at constant temperature. He also found that the presence of horseshoe vortices enhanced heat transfer due to better mixing in that region.

Mass transport studies for large Schmidt numbers for time-periodic flows past a cylinder are not readily available in the literature. This study focuses on pulsatile flow past a single cylinder and its effect on the velocity field and mass transfer at the cylinder surface. The parameter ranges investigated are $5 \leqslant \mathrm{Re} \leqslant 40$ and $\mathrm{Sc}=1000$.

\section{PROBLEM STATEMENT}

We consider flow of a Newtonian fluid, with viscosity $\mu$ and density $\rho$, past an infinitely long horizontal cylinder placed perpendicularly to an approaching unsteady stream in the $x$-axis direction, $u_{\infty}^{\prime}$, with concentration $C_{\infty}=0$ as shown in Fig. 1. The cylinder has radius $a$ and constant surface concentration $C_{S}$. A pulsatile free-stream velocity profile is considered, 


$$
u_{\infty}{ }^{\prime}=U_{o}\left[1+A \sin \left(\omega t^{\prime}\right)\right],
$$

where $U_{o}$ is the mean velocity, $A$ is the dimensionless amplitude of velocity oscillation, $\omega$ is the angular frequency of oscillation, and $t^{\prime}$ is time. Steady flow represents the special case of Eq. (1) when $A=0$. The flow is assumed to be twodimensional, and a polar coordinate system $(r, \theta)$ is used.

The conservation of momentum and mass equations can be expressed as

$$
\alpha^{2} \frac{\partial \underline{u}}{\partial t}+\frac{\operatorname{Re}}{2}(\underline{u} \cdot \underline{\nabla}) \underline{u}=-\underline{\nabla} p+\nabla^{2} \underline{u}
$$

and

$$
\underline{\nabla} \cdot \underline{u}=0
$$

where $\underline{u}=\left(u_{r}, u_{\theta}\right)$ is the velocity vector, $p$ is the pressure, and $\nabla$ is the gradient operator, in polar coordinates, $\nabla=\partial / \partial r \underline{e}_{r}$ $\overline{+} 1 / r \partial / \partial \theta \underline{e}_{\theta}$.

Thus, three dimensionless parameters control this flow: the oscillation amplitude, $A$; the Reynolds number, Re $=2 a U_{o} \rho / \mu$; and the Womersley number, $\alpha=a(\omega \rho / \mu)^{1 / 2}$. The variables used in the above conservation equations are all dimensionless and are related to their dimensional quantities (with primes) by

$$
\underline{u}=\frac{\underline{u}^{\prime}}{U_{o}}, \quad r=\frac{r^{\prime}}{a}, \quad(x, y)=\frac{\left(x^{\prime}, y^{\prime}\right)}{a}, \quad p=\frac{p^{\prime}}{\frac{\mu U_{o}}{a}},
$$

and

$$
t=\omega t^{\prime} \text {. }
$$

The boundary conditions on the surface of the cylinder $(r=1)$ are no slip and no penetration,

$$
u_{\theta}(r=1)=0
$$

and

$$
u_{r}(r=1)=0,
$$

respectively. Free-stream conditions are imposed far away from the cylinder $(r \rightarrow \infty)$,

$$
\underline{u} \cdot \underline{e}_{x}(r \rightarrow \infty)=1+A \sin (t)
$$

and

$$
\underline{u} \cdot \underline{e}_{y}(r \rightarrow \infty)=0 .
$$

The mass transport is governed by the convectiondiffusion equation,

$$
\alpha^{2} \mathrm{Sc} \frac{\partial C}{\partial t}+\frac{\mathrm{Re}}{2} \mathrm{Sc} \underline{\nabla} \cdot(\underline{u} C)=\nabla^{2} C
$$

where $C$ is the gas concentration. An additional dimensionless parameter becomes important, the Schmidt number, Sc $=\mu / \rho D$, where $D$ is the molecular diffusivity. The concentration in Eq. (8) has been scaled by the cylinder surface concentration,

$$
C=\frac{C^{\prime}}{C_{S}}
$$

The boundary condition on the surface of the cylinder $(r$ =1) is

$$
C(r=1)=1 .
$$

At the outer boundary $(r \rightarrow \infty)$, inflow-outflow conditions are prescribed,

$$
C(r \rightarrow \infty)=0, \quad \pi / 2<\theta<3 \pi / 2
$$

and

$$
\left.\frac{\partial C}{\partial r}\right|_{r \rightarrow \infty}=0, \quad-\pi / 2 \leqslant \theta \leqslant \pi / 2 .
$$

To simplify the problem further, the flow and concentration fields are assumed to be symmetric about $y=0$ for the range of Reynolds numbers investigated, $5 \leqslant \operatorname{Re} \leqslant 40$. This is consistent with previous experimental studies on a cylinder oscillating in a tank of fluid, ${ }^{19}$ and numerical studies of oscillating flow around a cylinder ${ }^{20,21}$ and pulsatile flow past a cylinder. ${ }^{15}$ In these studies, vortex shedding occurred in symmetric pairs and did not alter the flow-field symmetry. This can be contrasted with steady laminar flow about a cylinder in which three different regions exist: steady flow without separation $(\operatorname{Re} \lesssim 5)$, steady flow with two stationary symmetric vortices behind the cylinder $(5 \lesssim \operatorname{Re} \lesssim 46)$, and steady flow with asymmetric vortex shedding $(\operatorname{Re} \gtrsim 46) .^{22}$

The boundary conditions imposed at the symmetry line $(\theta=0, \pi)$ are

$$
\begin{gathered}
u_{\theta}(\theta=0, \pi)=0, \\
\left.\frac{\partial u_{r}}{\partial \theta}\right|_{(\theta=0, \pi)}=0,
\end{gathered}
$$

and

$$
\left.\frac{\partial C}{\partial \theta}\right|_{(\theta=0, \pi)}=0
$$

\section{NUMERICAL SOLUTION METHOD}

The SIMPLER method ${ }^{23}$ has been employed to numerically solve the coupled momentum and mass conservation equations, Eqs. (2) and (3). This method involves solving the velocity and pressure fields iteratively from an initial flow field. At each iteration, the algorithm consists of three steps: the solution of the momentum, pressure, and pressure correction equations, respectively.

The momentum equations in this method are obtained by integrating Eq. (2) over a control volume. The time derivative in Eq. (2) is approximated using a first-order implicit scheme. This leads to the following approximation:

$$
a_{C} \underline{u}_{C}=\sum a_{i} \underline{u}_{i}(i=E, W, N, S)+\underline{S}_{v}-\left.\underline{\nabla} p\right|_{C} .
$$

The coefficients $a$ contain some of the convective and diffusive flux terms and $a_{c}$ also contains part of the unsteady acceleration term. They are evaluated by means of a power- 


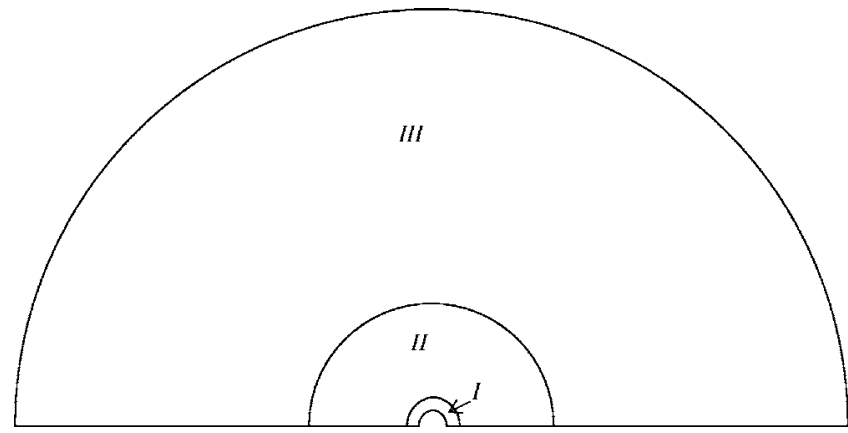

FIG. 2. Schematic of computational grid with three distinct regions.

law hybrid differencing scheme. ${ }^{23} S_{v}$ contains the remaining convective and unsteady acceleration terms, which are considered as known quantities evaluated from the previous iteration. A staggered grid is used where the velocity components are stored at $C$ and at the four neighboring points, $E$, $W, N$, and $S$, and the pressure is stored at $e$ and $w$, midway between $C$ and $E$, and $C$ and $W$, respectively, and at $n$ and $s$, midway between $C$ and $N$, and $C$ and $S$, respectively.

The pressure equation is obtained by substituting the momentum equations into the continuity equation to give

$$
\begin{aligned}
b_{c} p_{c}= & \sum b_{i} p_{i}(i=e, w, n, s)-\hat{u}_{\theta_{E}} \Delta r+\hat{u}_{\theta_{W}} \Delta r-\hat{u}_{r_{N}} r_{N} \Delta \theta \\
& +\hat{u}_{r_{S}} r_{S} \Delta \theta,
\end{aligned}
$$

where $e, w, n$, and $s$ are the pressure grid points neighboring $C$ and $\hat{u}_{i}$ represent temporary velocities defined by the momentum equation with the pressure term missing,

$$
\hat{\underline{u}}_{C}=\frac{1}{a_{C}}\left[\sum a_{i} \underline{u}_{i}(i=E, W, N, S)+\underline{S}_{v}\right] .
$$

The pressure correction equation accounts for the imbalance in mass in the control volume when the velocity field does not satisfy the continuity equation. It is obtained by substituting the following velocity correction equation into the continuity equation, Eq. (3):

$$
\underline{u}_{C}=\left[\underline{u}_{C}^{*}-\left.\frac{1}{a_{C}} \underline{\nabla} p^{*}\right|_{C}\right] \text {. }
$$

The pressure correction is very similar to the pressure equation, Eq. (16), as shown below,

$$
\begin{aligned}
b_{c} p_{c}^{*}= & \sum b_{i} p_{i}^{*}(i=e, w, n, s)-u_{\theta_{E}}^{*} \Delta r+u_{\theta_{W}}^{*} \Delta r-u_{r_{N}}^{*} r_{N} \Delta \theta \\
& +u_{r_{S}}^{*} r_{S} \Delta \theta
\end{aligned}
$$

the difference is that $p$ has been replaced with $p^{*}$ and $\hat{u}$ with $\underline{u}^{*}$, the predicted velocities.

In summary, for each time step the velocity and pressure fields are calculated according to the following algorithm: (1) The calculation starts from an assumed velocity profile; (2) the pressure equation, Eq. (16), is solved using the known temporary velocities, $\hat{u}$, Eq. (17); (3) the momentum equations, Eq. (15), are solved using the calculated pressure field in the previous step; (4) the pressure correction equation, Eq. (19), is solved using the predicted velocity field $\underline{u}^{*}$, which is the solution of the momentum equations obtained in the previous step; (5) the velocities are corrected with Eq. (18). The SuperLU solver ${ }^{24}$ is used to solve the linear systems in steps (2), (3), and (4). Steps (2)-(5) are repeated until convergence is achieved as defined by

$$
\max \left(\left\|\underline{u}^{N}-\underline{u}^{N-1}\right\|\right)<10^{-4}
$$

where $N$ is the number of iterations. Time is then incremented and the process described above, beginning with step (1), is repeated. The computation was terminated when periodic convergence was achieved. Periodic convergence was defined as

$$
\max [\|\underline{u}(\underline{r}, t)-\underline{u}(\underline{r}, t-2 \pi)\|]<10^{-3},
$$

the maximum difference in the entire velocity field for consecutive time cycles.

The mass transport equation can also be integrated over a control volume to give the approximation

$$
d_{C} C_{C}=\sum d_{i} C_{i}(i=e, w, n, s)+S_{c},
$$

where the concentration is stored at the pressure points. As for the momentum equations, the $d$ coefficients in Eq. (22) contain some of the convective and diffusive flux terms and are evaluated by means of a power-law hybrid differencing scheme; ${ }^{23} d_{c}$ also contains part of the unsteady acceleration

\begin{tabular}{|c|c|c|c|c|c|c|c|}
\hline & \multicolumn{4}{|c|}{ Number of points $(r \times \theta)$} & \multicolumn{3}{|c|}{ Radial domain } \\
\hline & Region I & Region II & Region III & Total & Region I & Region II & Region III \\
\hline Grid 1 & $75 \times 112$ & $190 \times 112$ & $56 \times 112$ & 35952 & 1 to 1.5 & 1.5 to 50 & 50 to 300 \\
\hline Grid 2 & $75 \times 112$ & $250 \times 112$ & $44 \times 112$ & 41328 & 1 to 1.5 & 1.5 to 100 & 100 to 300 \\
\hline Grid 3 & $75 \times 112$ & $190 \times 112$ & $65 \times 112$ & 36960 & 1 to 1.5 & 1.5 to 50 & 50 to 400 \\
\hline
\end{tabular}
term. $S_{c}$ contains the remaining unsteady acceleration term, which is based on quantities from the previous iteration. The concentration field is thus solved iteratively using the converged velocity field, where the linear system of equations for each iteration is solved using SuperLU. The same convergence criteria as listed above in Eqs. (20) and (21) were used with $\underline{u}$ replaced by $C$.

TABLE I. Numerical and geometric information for computational grids used in this study. 
(a)

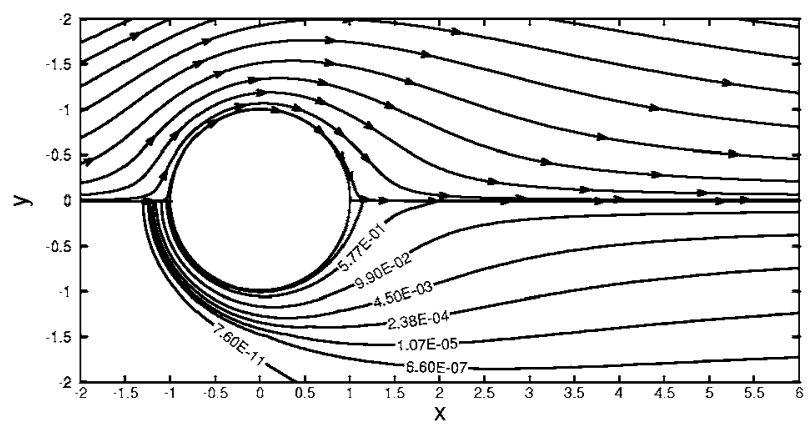

(b)

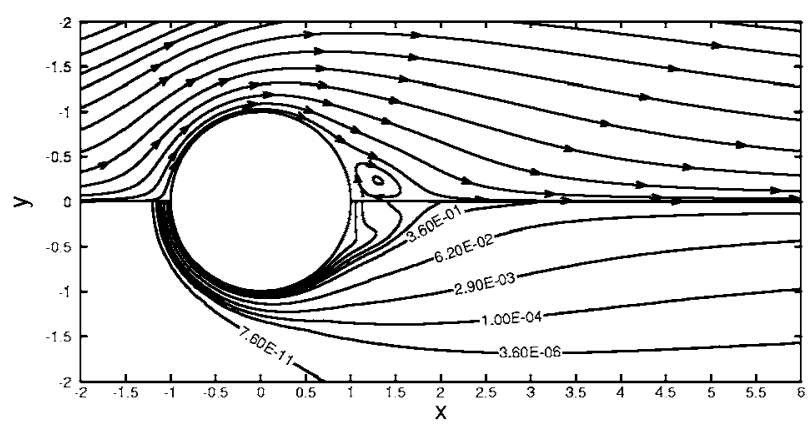

(c)

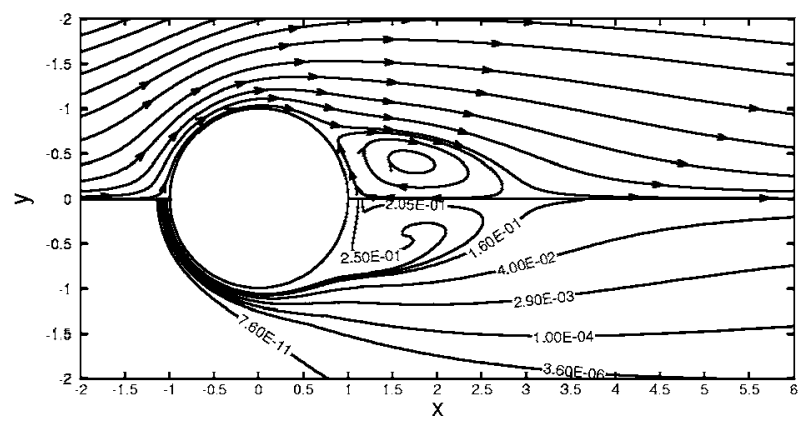

(d)

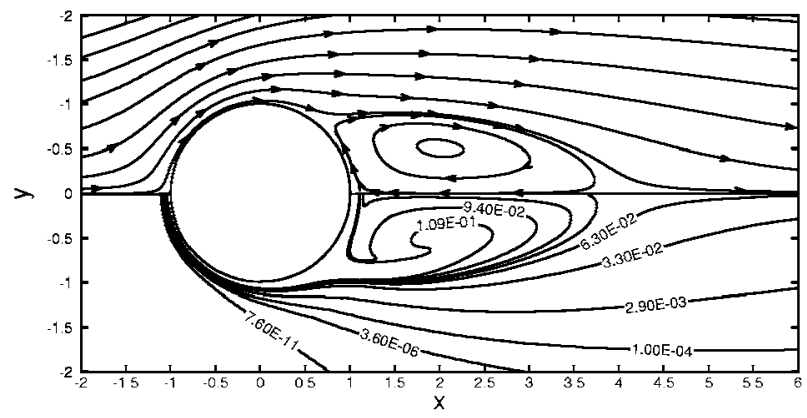

$(e)$

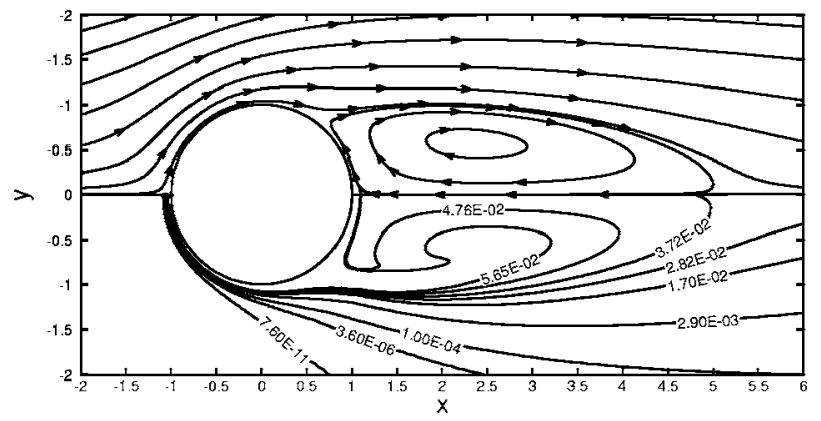

FIG. 3. Streamline and concentration fields $(\mathrm{Sc}=1000)$ for steady flow over a cylinder, top half and bottom half, respectively (a) $\mathrm{Re}=5$; (b) $\mathrm{Re}=10$; (c) $\operatorname{Re}=20$; (d) $\operatorname{Re}=30$; and (e) $\operatorname{Re}=40$.

Thus, the solution of the momentum equations and mass transport equation are decoupled and the fluid is assumed to have constant physical properties (i.e., density and viscosity), with Re based on the free-stream properties. In particular, the additional mass entering the free stream from the cylinder is relatively small as is its concentration in the free stream. Hence, its influence on the free-stream fluid is considered negligible. Studies investigating the influence of dissolved gases on liquid density show very little change in density as compared to gas-free liquids. ${ }^{25,26}$ Similar studies investigating the influence of dissolved gases on liquid viscosity are not readily available, but it is reasonable to assume that the change in viscosity due to dissolved gases would also be small. Consequently, the combination of the small quantity and concentration of dissolved gases in the fluid in our study, combined with the small change in physical properties due to dissolved gases, makes the assumption of constant properties in the free stream reasonable.

\section{COMPUTATIONAL DOMAIN}

In order to compute a numerical solution, it is necessary to define a specific location for the outer boundary, which will represent $r=\infty$. It was found that the region of flow influenced by the cylinder increased as Reynolds number decreased. This is consistent with the analysis of Lange et $a .^{22}$ for steady flow past a cylinder. They present recommended outer domain lengths for a range of Reynolds numbers to achieve an error less than $1 \%$ for the drag coefficient. This recommended outer boundary based on the smallest instantaneous Reynolds number $[\operatorname{Re}(1-A)]$ was used as a guideline. The actual outer boundary chosen was greater than this guideline and was tested to ensure that it did not influence the flow field. The computational domains used in this study range from $r=300$ to $r=400$. 
TABLE II. Comparison between the drag coefficient, $C_{D}$, obtained in this study and other numerical studies for the case of steady flow.

\begin{tabular}{ccccccc}
\hline \hline & \multicolumn{6}{c}{$C_{D}$} \\
\cline { 2 - 7 } & & $\begin{array}{c}\text { Hamielec and } \\
\text { Raul } \\
\text { (Ref. 28) }\end{array}$ & $\begin{array}{c}\text { Dennis and } \\
\text { Chang } \\
\text { (Ref. 27) }\end{array}$ & $\begin{array}{c}\text { D'Allesio and } \\
\text { Dennis } \\
\text { (Ref. 29) }\end{array}$ & $\begin{array}{c}\text { D'Allesio and } \\
\text { Denis } \\
\text { (Ref. 30) }\end{array}$ & $\begin{array}{c}\text { Lange } \text { et al. } \\
\text { (Ref. 22) }\end{array}$ \\
\hline 5 & 3.69 & $\ldots$ & 4.116 & 3.894 & 3.809 & 3.96 \\
10 & 2.62 & 2.75 & 2.846 & 2.719 & $\ldots$ & 2.76 \\
20 & 1.87 & $\ldots$ & 2.045 & 2.024 & 1.941 & 2.00 \\
30 & 1.59 & 1.588 & $\ldots$ & 1.673 & $\ldots$ & 1.69 \\
40 & 1.42 & $\ldots$ & 1.522 & 1.451 & 1.443 & 1.50 \\
\hline \hline
\end{tabular}

\section{GRID REFINEMENT AND NUMERICAL ACCURACY}

For the Reynolds numbers investigated, vortices appear at the rear of the cylinder. In addition, for large Schmidt numbers, a thin boundary layer for the concentration near the cylinder is expected. Consequently, a finer discretization of the grid near the cylinder and in the cylinder wake is required. Three different computational grids are used. In each grid, the computational domain is divided into three regions as shown in Fig. 2. In each of these regions, the grid is refined exponentially in the radial and angular directions to achieve grid refinement near $r=1$ and $\theta=0$, with each successive region becoming more coarse. The total number of points used ranges from $\sim 36000$ to $\sim 41500$. Specific details for the three computational grids are shown in Table I. It can be noted that grid 3 is merely an extension in the radial direction of grid 1 , and grid 2 radially extends region II of grid 1 . Grid 3 was used for all $\mathrm{Re}=5$ cases, grid 2 was used for $\operatorname{Re} \geqslant 10, \alpha \geqslant 2$, and $A=0.75$ cases (for which the vortex extended past $r=50$ during the cycle), and grid 1 was used for all other cases. All grids were tested for numerical accuracy by comparing the computed velocities and concentrations for a representative unsteady case to those obtained using grids additionally refined by $25 \%$ and $50 \%$ increases in grid points. It was found that the difference between the coarsest and finest grid was $<3 \%$ and that the difference between successively refined grids diminished. For the transient computations, a dimensionless time step of $2 \pi / 360$, corresponding to 360 time steps per cycle, was used and found to be sufficiently small to produce accurate solutions.

\section{RESULTS AND DISCUSSION}

The drag force, $F_{x}$, can be defined and calculated at the cylinder surface $(r=1)$ for any instant of time using

$$
\begin{aligned}
F_{x} & =\int_{\theta=0}^{\theta=2 \pi}\left[\left\{-p \underline{\underline{I}}+\left(\underline{\nabla} \underline{u}+(\underline{\nabla} \underline{u})^{T}\right)\right\} \cdot \underline{n}\right]_{r=1} \cdot \underline{e}_{x} d \theta \\
& =\int_{\theta=0}^{\theta=2 \pi}\left[\left(-p+2 \frac{\partial u_{r}}{\partial r} \mid\right)_{r=1} \cos \theta-\left.\frac{\partial u_{\theta}}{\partial r}\right|_{r=1} \sin \theta\right] d \theta,
\end{aligned}
$$

where $\underline{n}$ is the unit vector normal to the cylinder surface, and $\underline{e}_{x}$ is the unit vector in the $x$ direction.

A maximum drag force, $F_{x \text {-max }}$, can then be defined as

$$
F_{x-\max }=\max _{t}\left(F_{x}\right),
$$

which is the maximum $F_{x}$ during the time cycle.

In addition, a drag coefficient for any instant of time can be defined as

$$
C_{D}=\frac{2 F_{x}}{\operatorname{Re}}
$$

TABLE III. Comparison between the time averaged Sherwood number, $\overline{\overline{\mathrm{Sh}}}$, obtained in this study and those provided by other published predictive relations for the case of steady flow with $\mathrm{Sc}=1000$

\begin{tabular}{ccccccc}
\hline \hline & \multicolumn{5}{c}{$\overline{\mathrm{Sh}}$} \\
\cline { 2 - 7 } & & \multicolumn{3}{c}{$\begin{array}{c}\text { Kurdyumov } \\
\text { and } \\
\text { Re }\end{array}$} & $\begin{array}{c}\text { Van der } \\
\text { Filpert } \\
\text { (Ref. 9) }\end{array}$ & $\begin{array}{c}\text { Sparrow } \text { et al. } \\
\text { (Ref. 10) }\end{array}$ \\
\hline 5 & 13.43 & 16.93 & 13.80 & 14.85 & 14.12 & 14.03 \\
10 & 18.46 & 22.11 & 18.92 & 21.00 & $\ldots$ & 20.13 \\
20 & 27.37 & 28.87 & 26.18 & 29.69 & $\ldots$ & 28.99 \\
30 & 34.20 & 33.75 & 31.78 & 36.37 & $\ldots$ & 35.94 \\
40 & 39.40 & 37.70 & 36.52 & 42.00 & $\ldots$ & 41.88 \\
\hline \hline
\end{tabular}



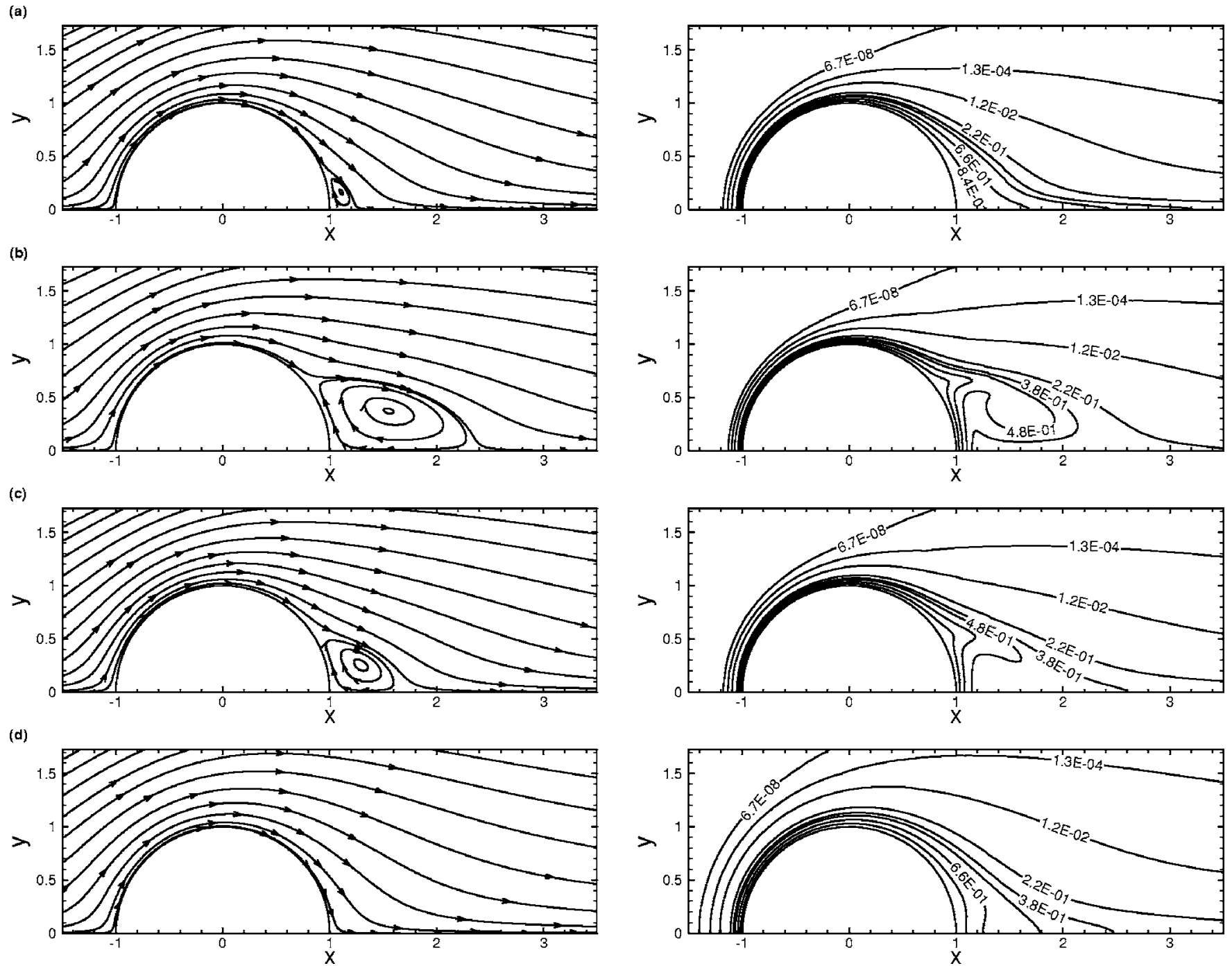

FIG. 4. Streamline and concentration field for $\mathrm{Re}=10, \alpha=0.25, A=0.75$, and $\mathrm{Sc}=1000$ plotted every quarter cycle (a) $t=0$; (b) $t=\pi / 2$; (c) $t=\pi$; and (d) $t=3 \pi / 2$.

Local, surface-averaged, and time-averaged Sherwood numbers, Sh, $\overline{\mathrm{Sh}}$, and $\overline{\overline{\mathrm{Sh}}}$, respectively, can also be defined on the cylinder surface $(r=1)$

$$
\begin{aligned}
& \operatorname{Sh}(\theta, t)=\frac{D(n \cdot \underline{\nabla} C)_{r^{\prime}=a}}{\frac{D\left(C_{S}-C_{\infty}\right)}{2 a}}=\left.2 \frac{\partial C}{\partial r}\right|_{r=1}, \\
& \overline{\operatorname{Sh}}(t)=\frac{1}{2 \pi} \int_{\theta=0}^{\theta=2 \pi} \operatorname{Sh} d \theta,
\end{aligned}
$$

and

$$
\overline{\overline{\mathrm{Sh}}}=\frac{1}{2 \pi} \int_{t=0}^{t=2 \pi} \overline{\mathrm{Sh}} d t .
$$

\section{A. Steady-state flow}

Prior to performing a parametric evaluation of the effect of amplitude and oscillation frequency on flow past and transport from a cylinder with an approaching pulsatile flow, a steady approaching flow is considered and the results are compared to those from other investigators. Figure 3 shows the streamline and concentration profiles for $\operatorname{Re}=5,10,20$, 30 , and 40 and $\mathrm{Sc}=1000$, where the streamlines are shown in the top half of the figure and the iso-concentration lines are shown in the bottom half. For $\mathrm{Re}=5$, Fig. 3(a), the flow remains attached to the cylinder and no separation or vortex is present at the rear of the cylinder; in addition, the isoconcentration lines behind the cylinder closely mirror the flow streamlines with the concentration decreasing with distance from the cylinder surface. For $\operatorname{Re}=10,20,30$, and 40, Figs. 3(b)-3(e), a pair of standing vortices exists behind the cylinder; the vortex size, length, and intensity increase as Reynolds number increases. Also, the angle of flow separation on the cylinder surface increases as Reynolds number increases. The streamline profiles in Fig. 3 are consistent with those published in other numerical studies. ${ }^{22,27}$ In terms of the concentration profile shown in Figs. 3(b)-3(e), the concentration gradient at the cylinder surface is smallest at the separation point where the mass is carried by the recir- 


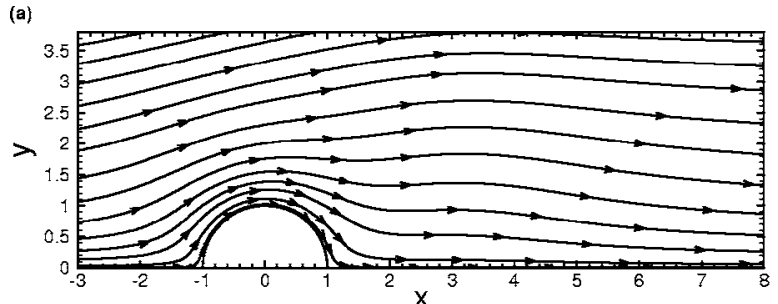

(b)

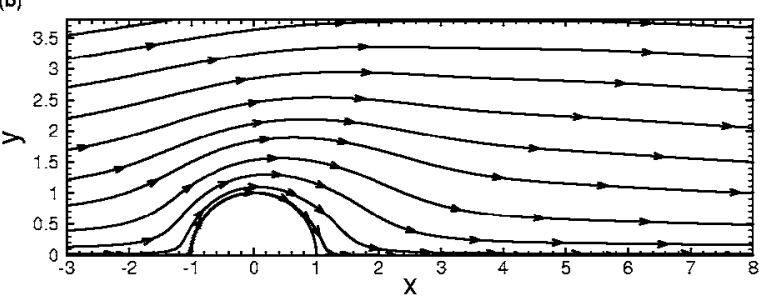

(c)

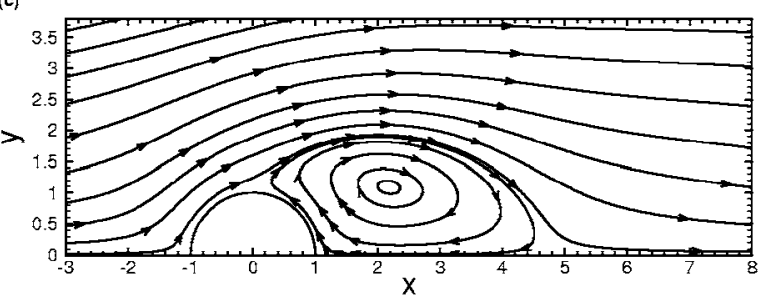

(d)

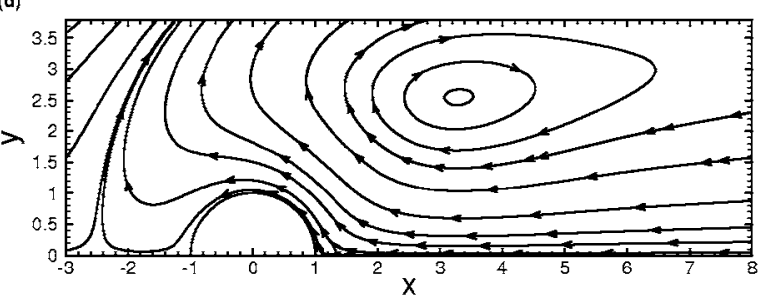

(1.)

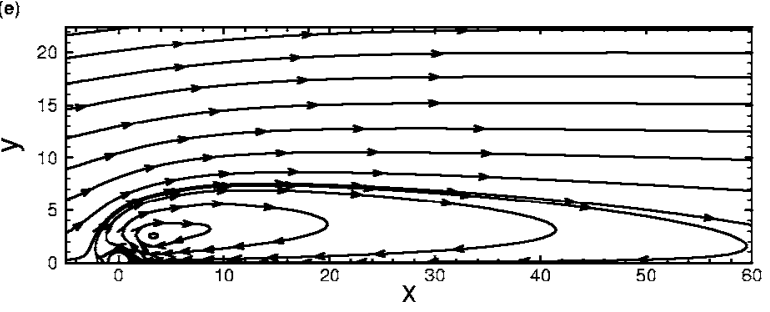

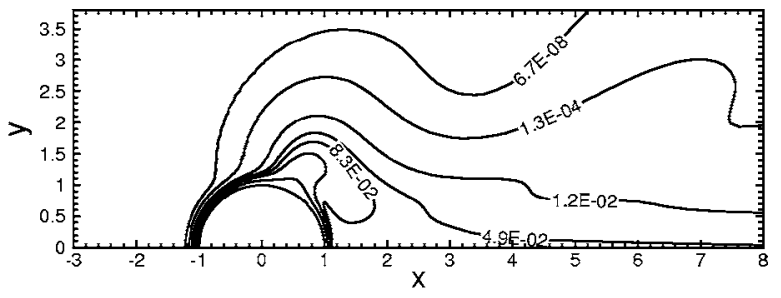
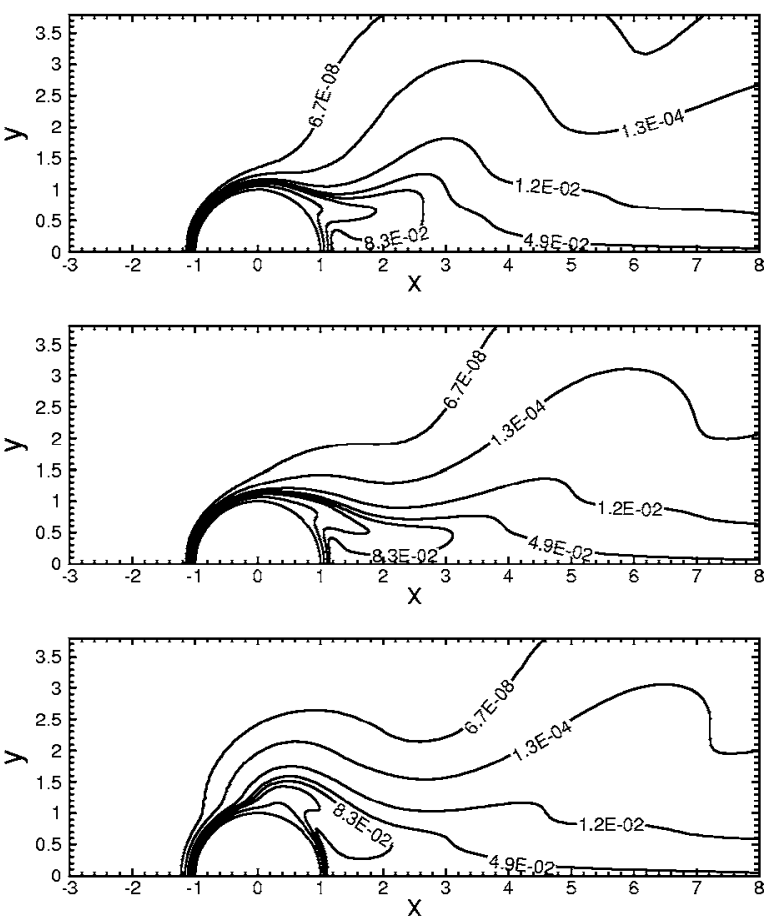

FIG. 5. Streamline and concentration field for $\mathrm{Re}=10, \alpha=2, A=0.75$, and $\mathrm{Sc}=1000$ plotted every quarter cycle (a) $t=0$; (b) $t=\pi / 2$; (c) $t=\pi$; (d) $t=3 \pi / 2$; and (e) expanded view for $t=3 \pi / 2$.

culation into the center of the vortex, thus creating a local concentration maximum. This local maximum decreases as Reynolds number increases.

A comparison of the drag coefficients calculated in the present study with other numerical results ${ }^{22,27-30}$ is shown in Table II. Good agreement is observed. From Table II, it is apparent that $C_{D}$ decreases as Re increases, though the actual drag force increases. An analysis of the individual components reveals that for small Reynolds numbers, the contribution of the pressure and viscous forces to the drag force is approximately equal. However, as Reynolds number increases, the pressure component of the drag force becomes more important.

Forced convection mass/heat transfer with constant concentration/temperature in laminar cross flow has been studied by many investigators and many correlations have been suggested. The calculated $\overline{\overline{\mathrm{Sh}}}$ values for the present in- vestigation are listed in Table III along with those provided by published predictive relations. Table III shows good agreement between the present and predicted results. It shows that as $\operatorname{Re}$ increases the increase in $\overline{\overline{\mathrm{Sh}}}$ is approximately proportional to $\mathrm{Re}^{1 / 2}$. From a boundary layer analysis for steady laminar flow past a cylinder, the typical finding is that most of the upstream side of the cylinder has a boundary layer which varies as $\mathrm{Re}^{-1 / 2}$, and on the downstream side of the cylinder the adverse pressure gradient forces the boundary layer to depart from the surface. Beyond the separation point, the boundary layer theory fails and there is no other simple explanation or theory. ${ }^{31}$ Some authors have considered a two-term $\mathrm{Nu}$ relationship, the first term proportional to $\mathrm{Re}^{1 / 2}$ for the upstream side of the cylinder and the second proportional to $\mathrm{Re}^{2 / 3}$ for the downstream side of the cylinder. ${ }^{32,33}$ Based on the assumption that most of the trans- 
(a)

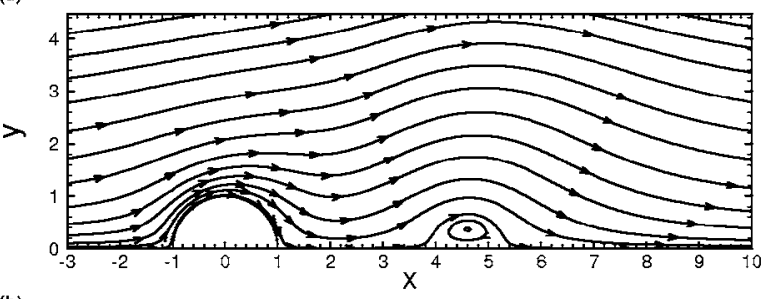

(b)

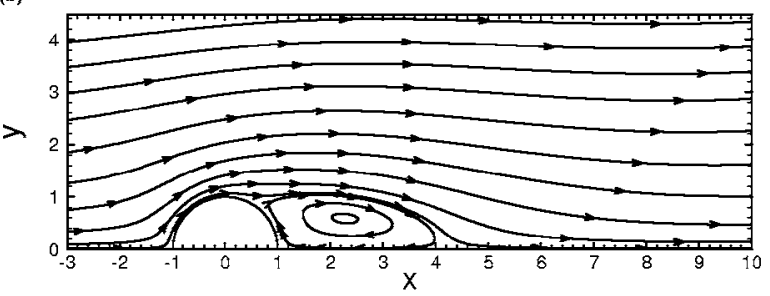

(c)

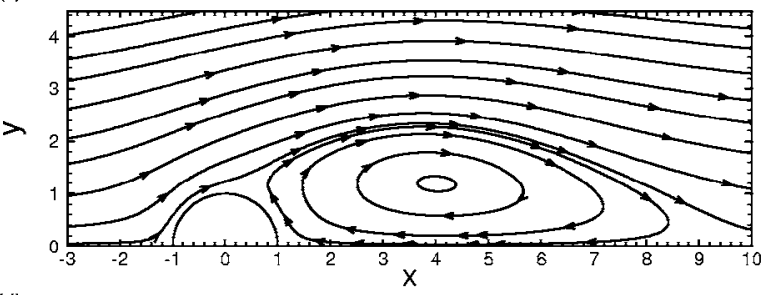

(d)

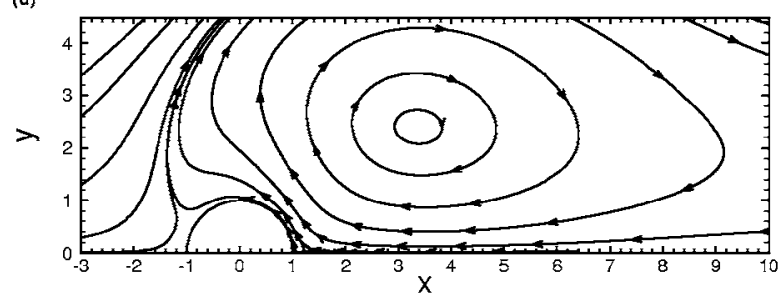

(e)

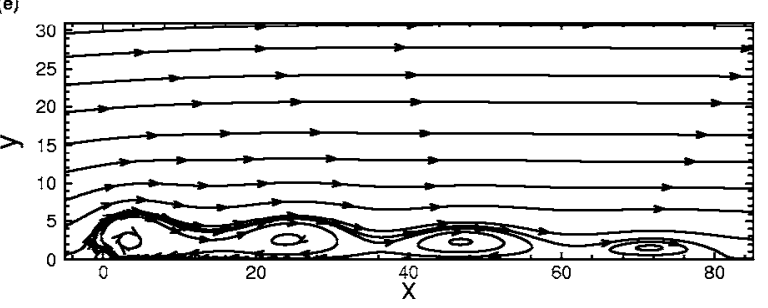

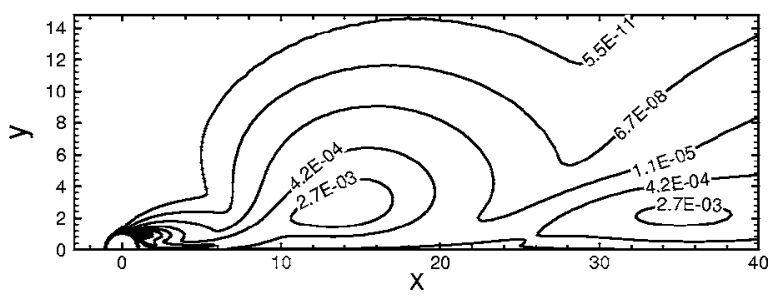
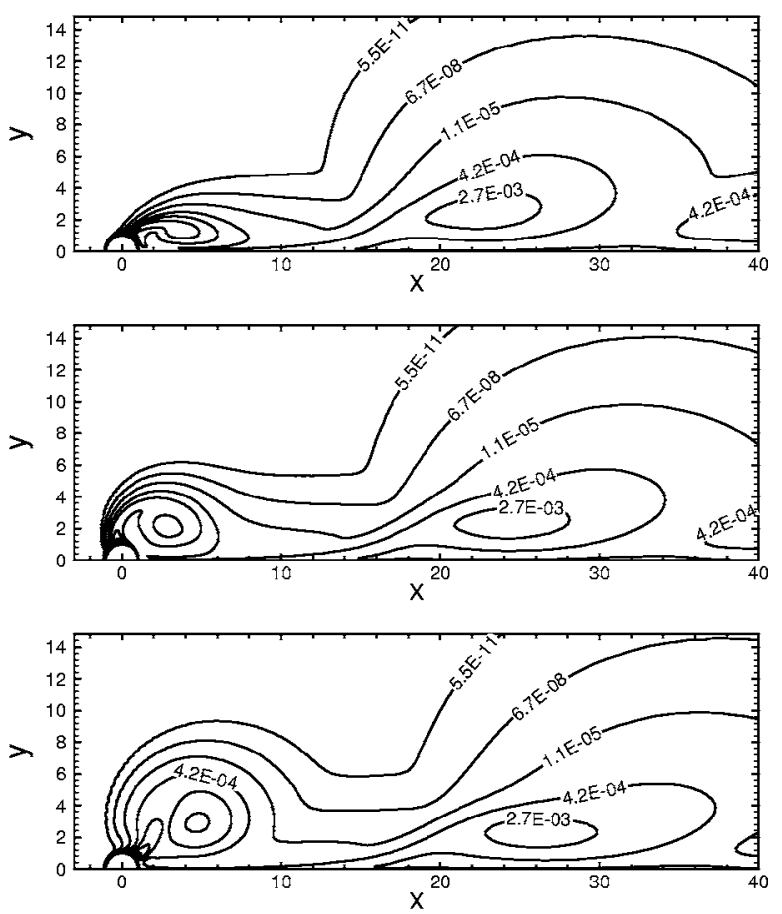

FIG. 6. Streamline and concentration field for $\mathrm{Re}=40, \alpha=2, A=0.75$, and $\mathrm{Sc}=1000$ plotted every quarter cycle (a) $t=0$; (b) $t=\pi / 2$; (c) $t=\pi$; (d) $t=3 \pi / 2$; and (e) expanded view for $t=3 \pi / 2$.

port occurs on the upstream side of the cylinder, it is reasonable to conclude that $\overline{\overline{\mathrm{Sh}}}$ should be approximately proportional to $\mathrm{Re}^{1 / 2}$ as observed in Table III. Unfortunately, correlations or data for local Sherwood numbers, Sh, corresponding to large Sc are not readily available in the literature to allow comparison with the present study.

\section{B. Pulsatile flow}

The influence of an approaching pulsatile flow on the streamline and concentration fields near a cylinder is investigated for $\mathrm{Re}=5,10,20,30$, and 40 and $\mathrm{Sc}=1000$ for all cases. The amplitudes considered were $A=0.25,0.5$, and 0.75 and the Womersley parameters considered were $\alpha$ $=0.25,2$, and 4 .

A detailed depiction of the streamline and concentration fields for $\operatorname{Re}=10, \alpha=0.25$, and $A=0.75$ is shown in Fig. 4 . Figure 4(b) shows the streamlines and iso-concentration pat- terns corresponding to maximum free-stream velocity, while Fig. 4(d) corresponds to minimum free-stream velocity. From Fig. 4, it can be seen that during one cycle the vortex oscillates in size while remaining attached to the cylinder. When the free-stream velocity is maximum at $t=\pi / 2, u_{\infty}$ $=u_{\max }=1+A$, the size of the vortex is greater and the enclosed vortical motion is stronger; when the free-stream velocity is minimum at $t=3 \pi / 2, u_{\infty}=u_{\min }=1-A$, the vortex has disappeared entirely. The oscillation in vortex size closely corresponds to the pulsation of the free-stream velocity. For the concentration field, the iso-concentration lines also grow and shrink in the wake region corresponding to the growth and reduction of the vortex.

The variation in the streamline pattern and isoconcentration pattern for $\operatorname{Re}=10, \alpha=2$, and $A=0.75$ is shown in Fig. 5. In this case, the vortex forms and begins to increase in size shortly after $u_{\infty}=u_{\max }$, Fig. 5(b). At $u_{\infty}=u_{\min }$, Figs. 
(a)

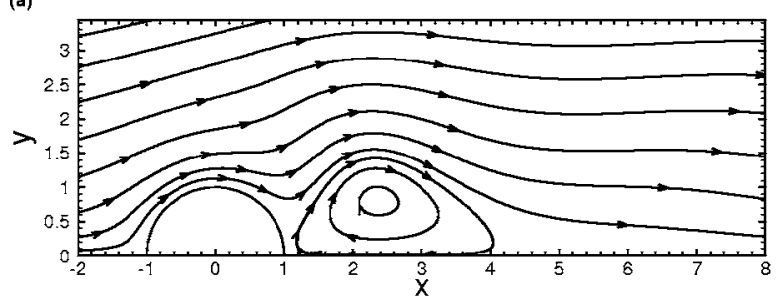

(b)

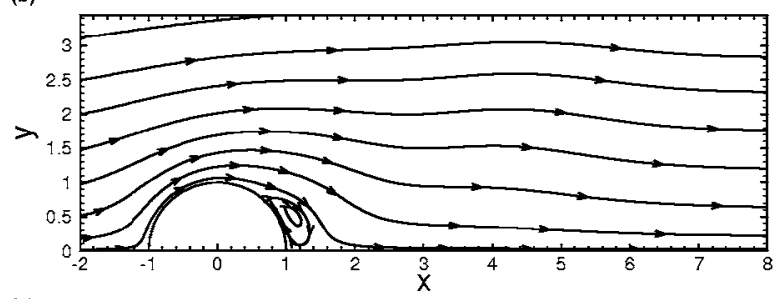

(c)

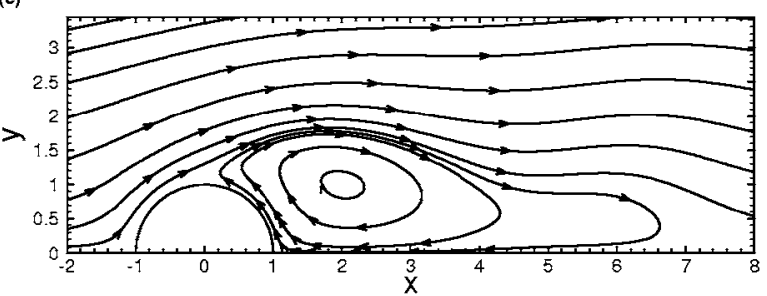

(d)

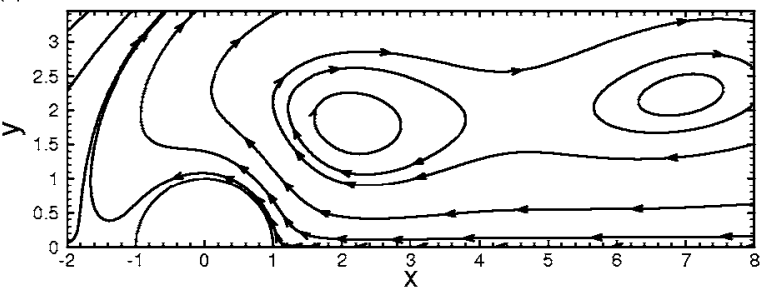

(e)

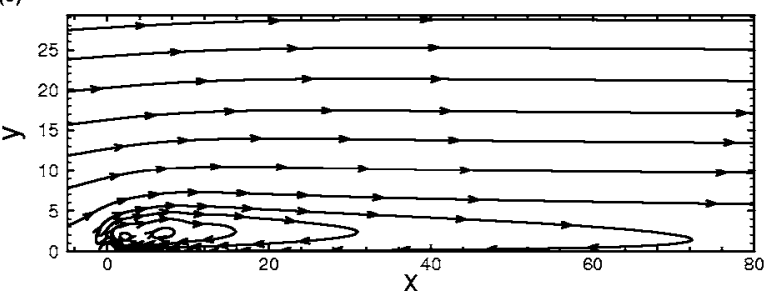

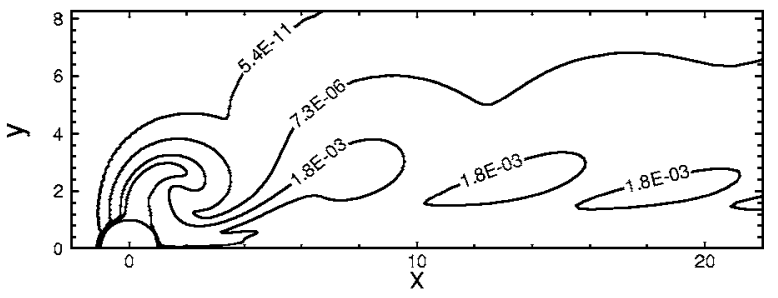
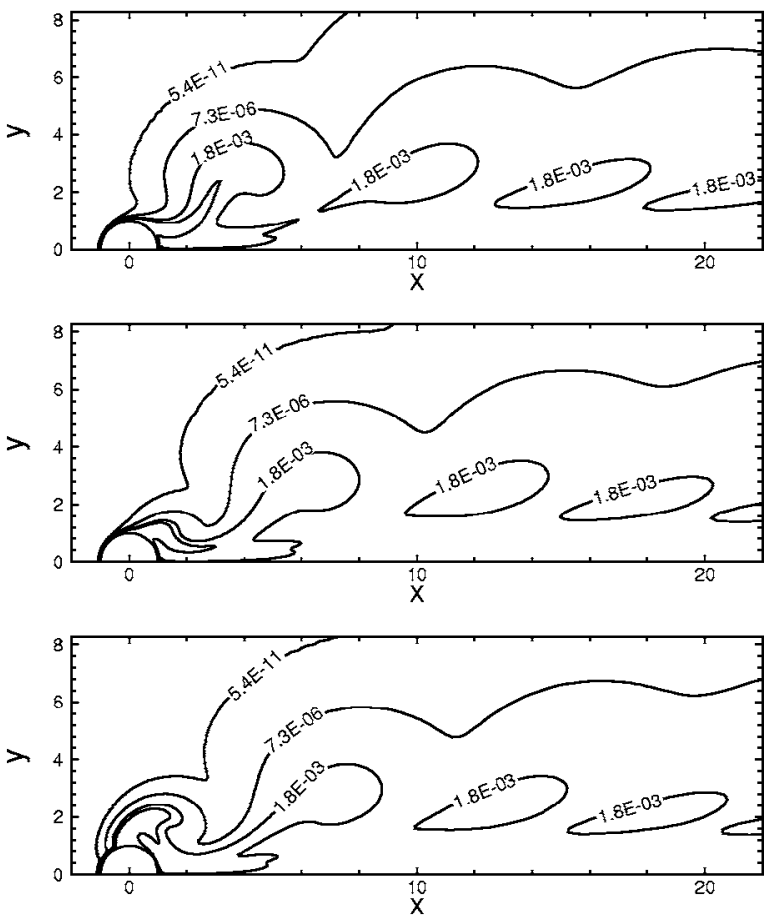

FIG. 7. Streamline and concentration field for $\mathrm{Re}=40, \alpha=4, A=0.75$, and $\mathrm{Sc}=1000$ plotted every quarter cycle (a) $t=0$; (b) $t=\pi / 2$; (c) $t=\pi$; (d) $t=3 \pi / 2$; and (e) expanded view for $t=3 \pi / 2$.

5(d) and 5(e), it is much larger, has been propagated far downstream, and has completely engulfed the cylinder with vortical motion significantly weaker. Shortly afterward, the vortex proceeds to rapidly decrease in size, detach from the cylinder, and disappear before $u_{\infty}=1$ at $t=0$, Fig. 5(a), where only traces of the detached and decayed vortex can be observed. The concentration field in the cylinder wake also oscillates during the cycle, being carried downstream when $u_{\infty} \gtrsim 1$ and carried upstream when $u_{\infty} \lesssim 1$.

The effect of a larger Reynolds number on the streamline and concentration fields is shown in Fig. 6 for $\operatorname{Re}=40, \alpha$ $=2$, and $A=0.75$. In Fig. 6(a) (corresponding to $t=0$ and $u_{\infty}$ $=1$ ), a vortex has recently been shed from the cylinder surface and is in the process of decaying. A new vortex then forms near the rear stagnation point and continues to grow during the latter half of acceleration and during deceleration, Figs. 6(b)-6(e). At $t=3 \pi / 2$ [Figs. 6(d) and 6(e)], four vorti- ces are observed inside the larger vortex. The movement of high momentum fluid induces an adverse pressure gradient which causes flow reversal of low momentum fluid near the symmetry line and the creation of additional vortices. During this process, each additional downstream vortex is sequentially formed and incorporated into the larger vortex structure as it continues to grow before the next additional vortex is formed. Each additional vortex is weaker in strength than the previous vortex. This process occurs during the latter half of deceleration. The additional vortices then sequentially diminish and disappear, starting with the vortex farthest downstream, until only the original vortex remains and is subsequently shed from the cylinder surface. The variation in the iso-concentration patterns is also shown in Figs. 6(a)-6(d). The increased Reynolds number greatly aids in the downstream propagation of mass from the cylinder surface; consequently, a larger region is influenced. Mass from the cyl- 


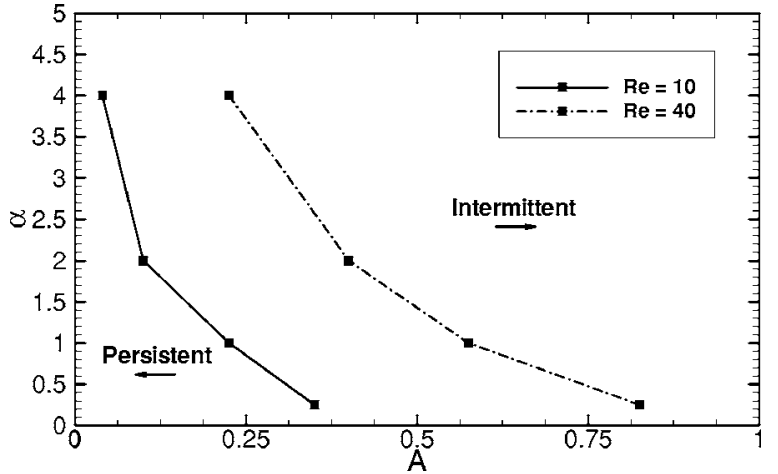

FIG. 8. Parameter space investigation for $\mathrm{Re}=10$ and $\mathrm{Re}=40$ showing spatial regions where a persistent vortex exists, present during the entire time cycle (left of curve), and where an intermittent vortex exists, present only during part of a time cycle (right of curve).

inder surface is swept downstream in waves, with each cycle releasing a small pocket of higher concentration fluid when $u_{\infty} \cong u_{\max }$, Fig. 6(b). The effect of approximately two cycles is shown in Fig. 6.

The streamline and concentration fields for $\operatorname{Re}=40, \alpha$ $=4$, and $A=0.75$ are shown in Fig. 7. Figure 7(a) (corresponding to $t=0$ and $u_{\infty}=1$ ) shows a vortex, which is larger in size than the cylinder, in the process of being shed. At $t$ $=\pi / 2$ when $u_{\infty}=u_{\max }$, a new vortex is being formed as shown in Fig. 7(b). This vortex continues to grow in size and its tail is simultaneously being swept downstream during the deceleration phase of the cycle. Eventually, a secondary vor-
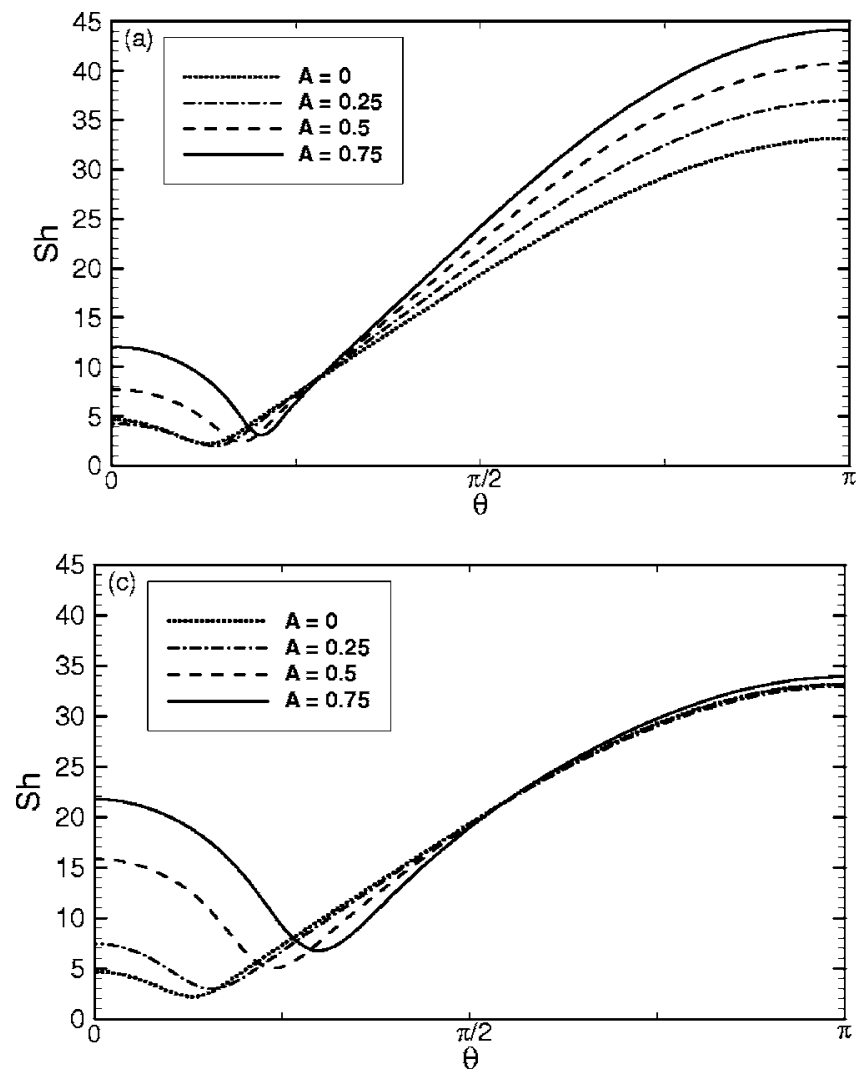

tex is formed downstream of the first in the tail of the primary vortex as shown in Figs. 7(d) and 7(e) when $u_{\infty}=u_{\min }$; a saddle point is thus created. This secondary vortex is weaker in strength than the primary vortex, and the entire vortex structure extends far downstream from the cylinder, which is enveloped by the vortex structure. During the acceleration phase, the vortices decrease in size and the secondary vortex collapses into the primary vortex, which is of increased strength, before later being shed from the cylinder in Fig. 7(a). The iso-concentration fields are also shown in Figs. $7(\mathrm{a})-7(\mathrm{~d})$. The pattern is very similar to that observed for $\operatorname{Re}=40, \alpha=2$, and $A=0.75$ in Fig. 7, with mass from the cylinder surface being propagated downstream in waves. A larger Womersley number results in the pockets of higher concentration fluid being released with greater frequency from the cylinder surface. The effect of approximately three cycles is shown in Fig. 7.

The presence of multiple vortices was also observed by $\operatorname{Badr}^{15}$ for pulsatile flow with $\mathrm{Re}=500, \mathrm{St}=\pi / 4$ (or $\alpha \cong 14$ ), and $A=0.5$, where $S t$ is the Strouhal number. However, the physics behind the vortices formation in that study is quite different from the present study. In his study, convection dominated the flow and two vortices were sequentially initiated at the cylinder surface before being individually propagated downstream. Thus, two vortices were formed and shed during each cycle. When $u_{\infty}=u_{\min }$, the two vortices are enveloped by a larger vortex structure which partially extends over the top of the cylinder; this flow structure is similar to that observed in the present study for $\operatorname{Re}=40, \alpha=2$, and $A$
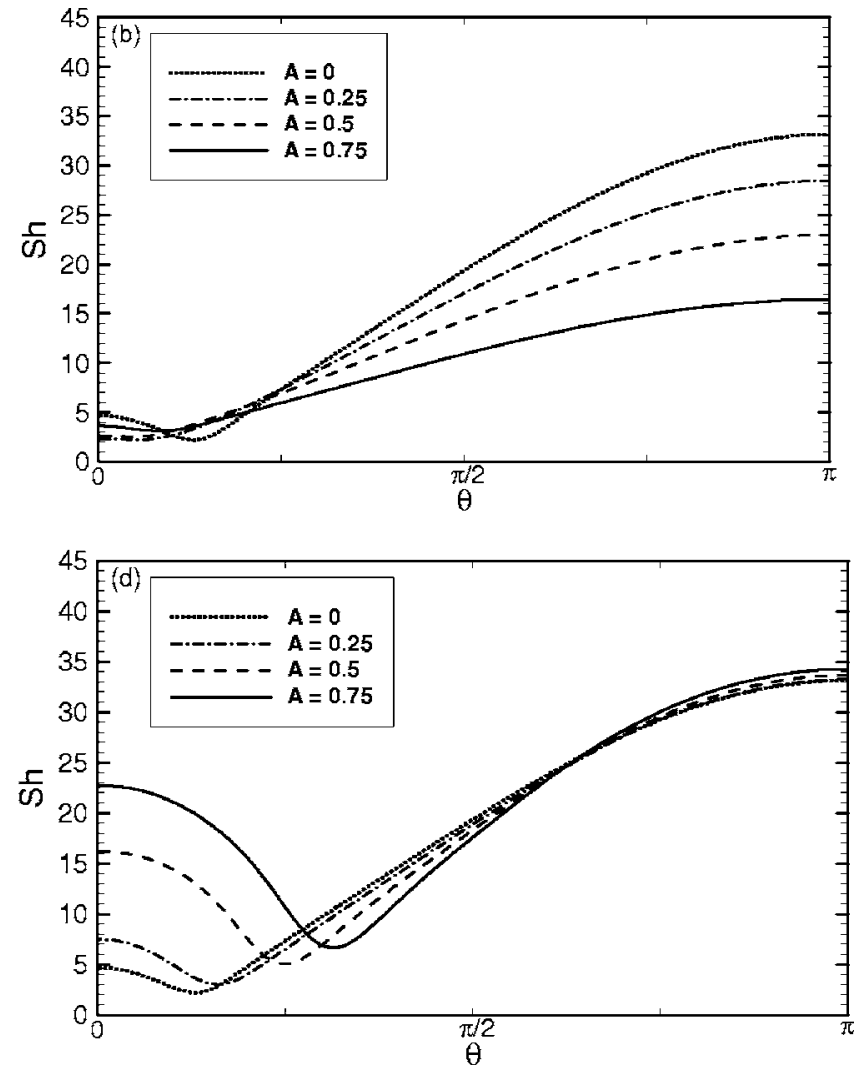

FIG. 9. The effect of amplitude of pulsation on the local Sherwood number for Re=10 and $\mathrm{Sc}=1000$ (a) $\alpha=0.25, t=\pi / 2 ;$ (b) $\alpha=0.25, t=3 \pi / 2 ;$ (c) $\alpha=2$, $t=\pi / 2 ;$ and (d) $\alpha=2, t=3 \pi / 2$. 

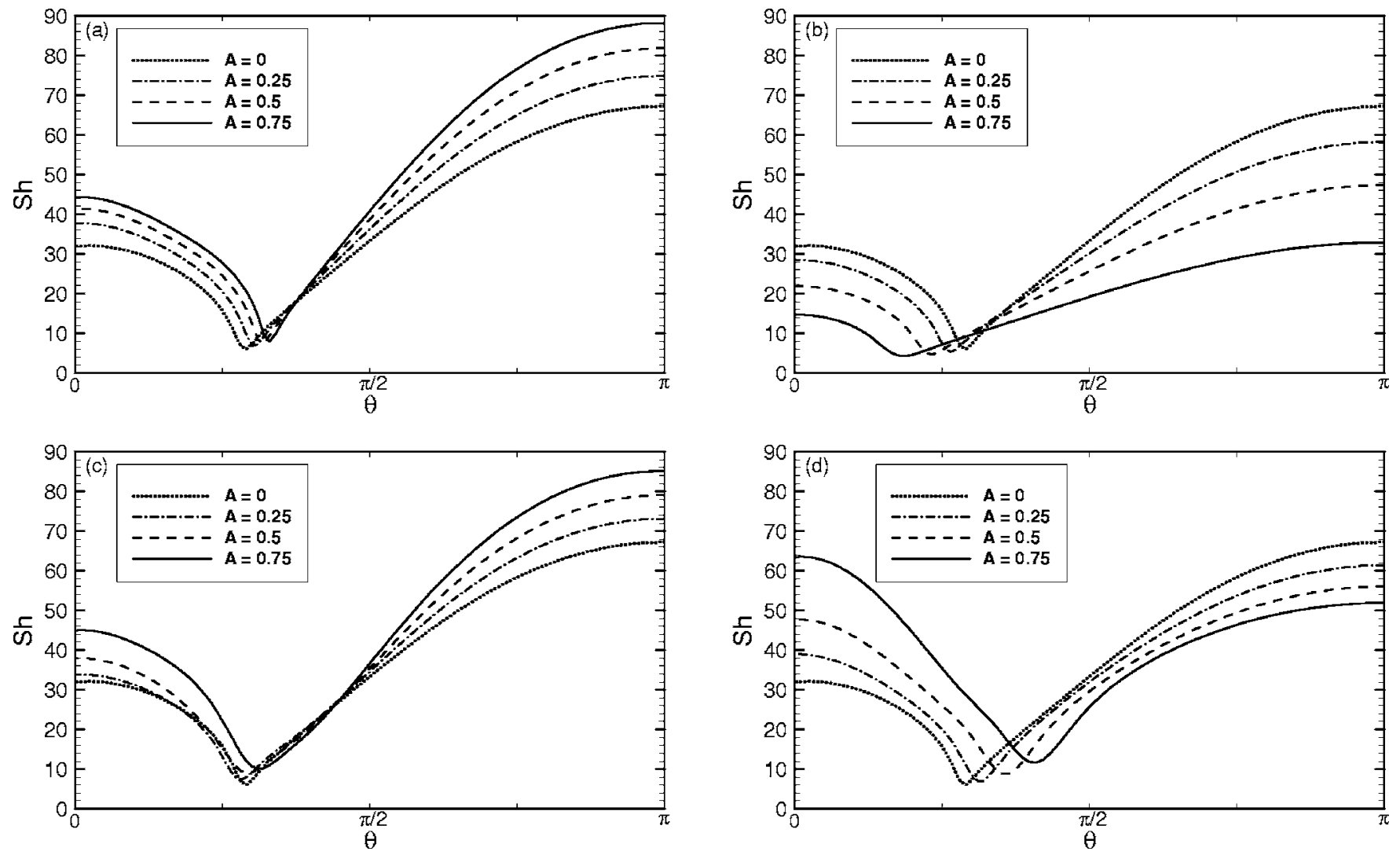

FIG. 10. The effect of amplitude of pulsation on the local Sherwood number for $\operatorname{Re}=40$ and $\mathrm{Sc}=1000$ (a) $\alpha=0.25, t=\pi / 2$; (b) $\alpha=0.25, t=3 \pi / 2$; (c) $\alpha=2$, $t=\pi / 2 ;$ and (d) $\alpha=2, t=3 \pi / 2$.

$=0.75$ [Fig. 6(c)] and for $\operatorname{Re}=40, \alpha=4$, and $A=0.75$ [Fig. $7(\mathrm{c})]$ when $u_{\infty}=u_{\text {min }}$. However, in his study, for the majority of the cycle the two vortices are distinct and separately grow and diminish before being shed, whereas in the present study, multiple vortices are observed only when the free-stream velocity is near $u_{\infty}=u_{\min }$ and the multiple vortices are always inside a larger vortex structure.

A parameter space analysis can be performed to better characterize the flow. For $\mathrm{Re}=10,20$, 30, and 40, a vortex exists downstream of the cylinder and it oscillates in size during one time cycle; these oscillations are governed by the Womersley parameter and the oscillation amplitude. In particular, a region of space where a vortex is attached to the cylinder throughout the cycle and a region of space where the vortex is intermittently attached to the cylinder during the cycle can be defined as shown in Fig. 8 for $\mathrm{Re}=10$ and $\mathrm{Re}$ $=40$, with the demarcation curve for the critical $\alpha$ defined by $\alpha_{c}=0.005 A^{-1.13} \operatorname{Re}^{1.33}$. For $\alpha<\alpha_{c}$, the vortex is always attached to the cylinder (persistent), while for $\alpha>\alpha_{c}$, the vortex is attached to the cylinder only during part of a cycle (intermittent). As Re decreases, the demarcation curve shifts to the left. Although not shown, a similar analysis is even applicable for $\operatorname{Re}=5$, for which no vortex is present in the steady-state solution. The effect of pulsation, however, induces a vortex during part of the cycle for almost all of the combinations of $\alpha$ and $A$ considered except the smallest, $\alpha$ $=0.25$ and $A=0.25$. Thus, two regions would exist for Re $=5$, an intermittent region (to the right of the curve) and a region where the flow remains attached to the cylinder without the presence of a vortex (to the left of the curve).

The effect of amplitude of pulsation on the local Sherwood number, $\mathrm{Sh}$, is shown for $\mathrm{Re}=10$ in Fig. 9 and for $\operatorname{Re}=40$ in Fig. 10 for half of the cylinder surface $(\theta=0-\pi$ where $\theta=0$ defines the rear stagnation point and $\theta=\pi$ defines the front stagnation point). The steady-state case, $A=0$, is also shown. Figures 9(a) and 9(c) correspond to maximum free-stream velocity, $u_{\infty}=u_{\max }$ when $t=\pi / 2$, and Figs. 9(b) and $9(\mathrm{~d})$ correspond to minimum free-stream velocity, $u_{\infty}=u_{\min }$ when $t=3 \pi / 2$. For $\operatorname{Re}=10$ and $\alpha=0.25$, when $t$ $=\pi / 2$ [Fig. 9(a)], Sh increases as the amplitude increases and is higher than the steady-state value on almost all of the surface; when $t=3 \pi / 2$ [Fig. 9(b)], Sh decreases as the amplitude decreases and is always lower than the steady-state value on almost all of the surface. This same phenomenon is observed for $\operatorname{Re}=40$ and $\alpha=0.25$ in Figs. 10(a) and 10(b) and is consistent with a quasi-steady-state explanation in which the higher/lower stream velocities lead to higher/lower velocity and concentration gradients. For $\operatorname{Re}=10$ and $\alpha=2$, the local Sherwood number distributions for $t=\pi / 2$ [Fig. $9(\mathrm{c})]$ and $t=3 \pi / 2$ [Fig. 9(d)] are approximately the same. In these figures, Sh is always higher than the steady-state value at the rear part of the surface, where the vortex is attached to the cylinder, and Sh increases with amplitude. At the front of the cylinder, Sh approaches approximately the same value irrespective of amplitude. In contrast, for $\operatorname{Re}=40$ and $\alpha=2$, the local Sherwood number distributions for $t=\pi / 2$ [Fig. 

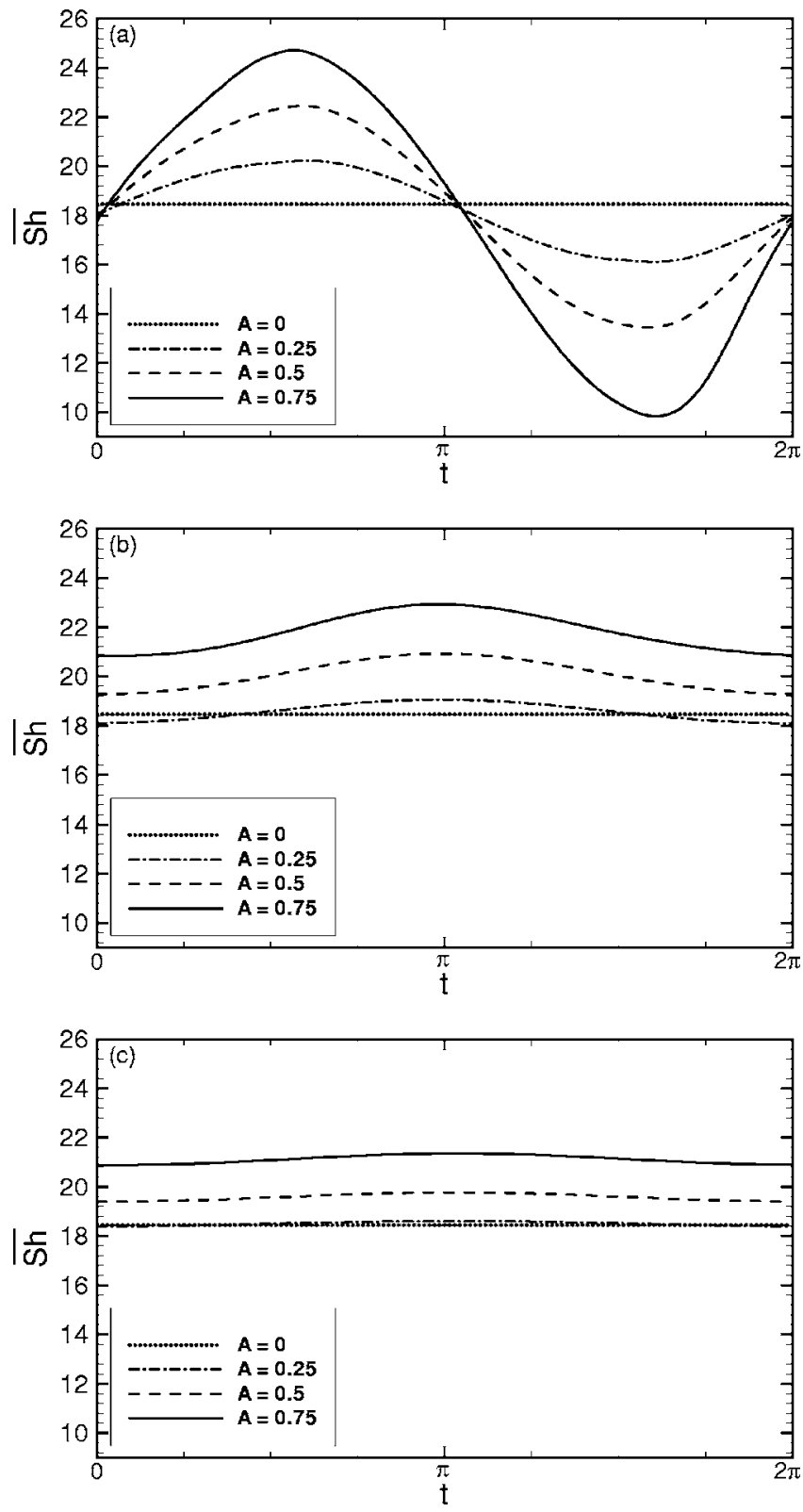

FIG. 11. The effect of amplitude of pulsation on the surface averaged Sherwood number for one time cycle for $\mathrm{Re}=10$ and $\mathrm{Sc}=1000$ (a) $\alpha=0.25$; (b) $\alpha=2$; and (c) $\alpha=4$.

10(c)] and $t=3 \pi / 2$ [Fig. 10(d)] are quite different. In Fig. 10 (c), Sh is higher than the steady-state value on almost all of the surface and increases with amplitude; this is consistent with the higher stream velocities which are present. In Fig. 10(d), Sh is higher than the steady-state value at the rear part of the surface, increasing with amplitude, and lower than the steady-state value at the front of the surface, decreasing with amplitude. When $t=3 \pi / 2$ [Fig. 10(d)], the difference between Sh near the rear and front stagnation points becomes smaller as amplitude increases; thus, transport near these points is of approximately equal importance. In fact, for Re $=40, \alpha=4$, and $A=0.75$, Sh is actually higher at the rear stagnation point instead of at the front stagnation point.

The effect of amplitude of pulsation on the surfaceaveraged Sherwood number, $\mathrm{Sh}$, during one time cycle is
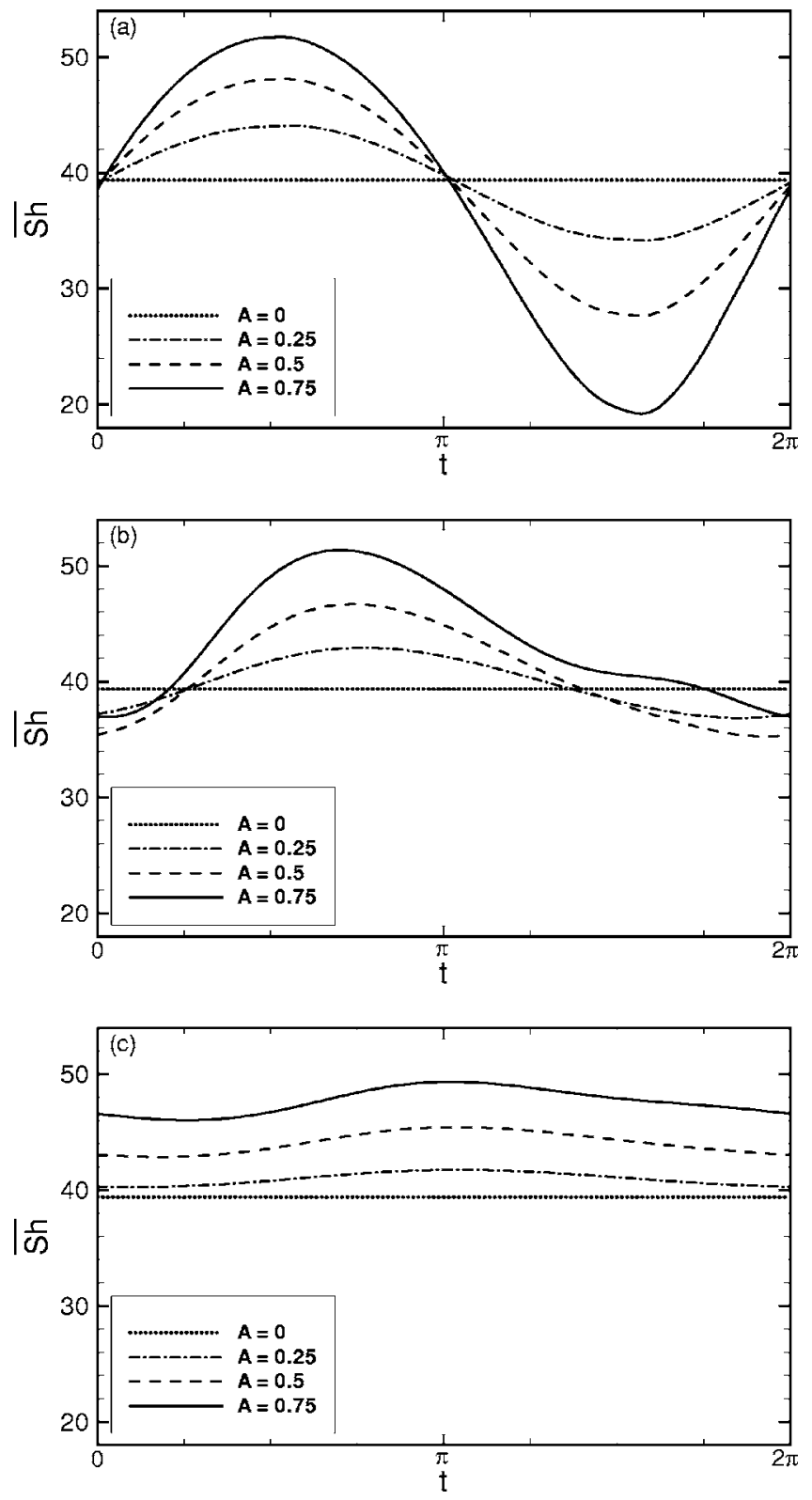

FIG. 12. The effect of amplitude of pulsation on the surface averaged Sherwood number for one time cycle for $\mathrm{Re}=40$ and $\mathrm{Sc}=1000$ (a) $\alpha=0.25$; (b) $\alpha=2$; and (c) $\alpha=4$.

shown for $\mathrm{Re}=10$ and $\mathrm{Re}=40$ in Figs. 11 and 12, respectively. The steady-state case, $A=0$, is also shown in the figures. For $\alpha=0.25$ and both $\operatorname{Re}=10$ and $\operatorname{Re}=40$, as shown in Figs. 11(a) and 12(a), respectively, the oscillation in $\overline{\mathrm{Sh}}$ nearly corresponds to the free-stream velocity pulsation. This is consistent with the previous quasi-steady-state explanation in which the higher/lower instantaneous free-stream velocities lead to higher/lower velocity and concentration gradients and correspondingly higher/lower instantaneous $\overline{\mathrm{Sh}}$ values. In contrast, for $\alpha=2$ and $\alpha=4$ in Figs. 11(b), 11(c), 12(b), and 12(c), the oscillation in Sh has a phase lag behind the pulsation in the free-stream velocity. This phase lag approaches approximately $\pi / 2$ as Womersley number increases irrespective of Reynolds number as shown in Figs. 11(b), 11(c), and 12(c). This approach to a phase lag of about $\pi / 2$ is faster for smaller Reynolds numbers as shown by compar- 
TABLE IV. The effect of a pulsatile free-stream velocity on the maximum drag force, $F_{x-\max }$, the time-averaged Sherwood number, $\overline{\overline{\mathrm{Sh}}}(\mathrm{Sc}=1000)$, and the vortex structure.

\begin{tabular}{|c|c|c|c|c|c|}
\hline $\mathrm{Re}$ & $\alpha$ & A & $F_{x-\max }$ & $\overline{\overline{\mathrm{Sh}}}$ & $\begin{array}{c}\text { Vortex } \\
\text { structure }^{a}\end{array}$ \\
\hline 5 & 0 & 0 & 9.22 & 13.56 & NV \\
\hline 5 & 0.25 & 0.25 & 12.84 & 13.52 & $\mathrm{NV}$ \\
\hline 5 & 0.25 & 0.5 & 16.95 & 13.30 & IN \\
\hline 5 & 0.25 & 0.75 & 21.40 & 12.89 & IN \\
\hline 5 & 2 & 0.25 & 20.86 & 13.51 & IN \\
\hline 5 & 2 & 0.5 & 32.65 & 13.39 & IN \\
\hline 5 & 2 & 0.75 & 44.40 & 14.03 & IN \\
\hline 5 & 4 & 0.25 & 43.49 & 13.53 & IN \\
\hline 5 & 4 & 0.5 & 77.93 & 13.53 & IN \\
\hline 5 & 4 & 0.75 & 112.44 & 13.67 & IN \\
\hline 10 & 0 & 0 & 13.02 & 18.46 & PE \\
\hline 10 & 0.25 & 0.25 & 18.22 & 18.22 & $\mathrm{PE}$ \\
\hline 10 & 0.25 & 0.5 & 24.25 & 18.14 & IN \\
\hline 10 & 0.25 & 0.75 & 30.86 & 17.82 & IN \\
\hline 10 & 2 & 0.25 & 24.52 & 18.58 & IN \\
\hline 10 & 2 & 0.5 & 36.17 & 20.07 & IN \\
\hline 10 & 2 & 0.75 & 48.04 & 21.80 & IN \\
\hline 10 & 4 & 0.25 & 46.97 & 18.51 & IN \\
\hline 10 & 4 & 0.5 & 81.09 & 19.60 & IN \\
\hline 10 & 4 & 0.75 & 115.58 & 21.12 & IN \\
\hline 20 & 0 & 0 & 18.79 & 27.37 & PE \\
\hline 20 & 0.25 & 0.25 & 26.69 & 26.94 & PE \\
\hline 20 & 0.25 & 0.5 & 35.72 & 26.64 & $\mathrm{PE}$ \\
\hline 20 & 0.25 & 0.75 & 45.74 & 26.05 & IN \\
\hline 20 & 2 & 0.25 & 31.51 & 27.97 & IN \\
\hline 20 & 2 & 0.5 & 44.70 & 29.59 & IN \\
\hline 20 & 2 & 0.75 & 58.59 & 31.67 & IN \\
\hline 20 & 4 & 0.25 & 52.15 & 28.01 & IN \\
\hline 20 & 4 & 0.5 & 85.58 & 30.01 & IN \\
\hline 20 & 4 & 0.75 & 119.51 & 32.53 & IN \\
\hline 30 & 0 & 0 & 23.72 & 34.20 & $\mathrm{PE}$ \\
\hline 30 & 0.25 & 0.25 & 33.97 & 33.83 & PE \\
\hline 30 & 0.25 & 0.5 & 45.68 & 33.30 & $\mathrm{PE}$ \\
\hline 30 & 0.25 & 0.75 & 58.74 & 32.29 & PE \\
\hline 30 & 2 & 0.25 & 38.70 & 34.69 & IN \\
\hline 30 & 2 & 0.5 & 54.49 & 35.89 & IN \\
\hline 30 & 2 & 0.75 & 72.04 & 38.32 & IN \\
\hline 30 & 4 & 0.25 & 56.61 & 35.21 & IN \\
\hline 30 & 4 & 0.5 & 89.43 & 37.85 & IN \\
\hline 30 & 4 & 0.75 & 122.82 & 41.00 & IN \\
\hline 40 & 0 & 0 & 28.14 & 39.40 & $\mathrm{PE}$ \\
\hline 40 & 0.25 & 0.25 & 40.62 & 39.30 & PE \\
\hline 40 & 0.25 & 0.5 & 54.78 & 38.64 & $\mathrm{PE}$ \\
\hline 40 & 0.25 & 0.75 & 70.64 & 37.42 & $\mathrm{PE}$ \\
\hline 40 & 2 & 0.25 & 45.62 & 39.88 & PE \\
\hline 40 & 2 & 0.5 & 64.42 & 40.79 & IN \\
\hline 40 & 2 & 0.75 & 85.55 & 43.63 & IN \\
\hline 40 & 4 & 0.25 & 66.64 & 40.99 & IN \\
\hline 40 & 4 & 0.5 & 93.29 & 44.10 & IN \\
\hline 40 & 4 & 0.75 & 125.85 & 47.65 & IN \\
\hline
\end{tabular}

${ }^{\mathrm{a}} \mathrm{NV}=$ No vortex, $\mathrm{PE}=$ persistent vortex, and $\mathrm{IN}=$ intermittent vortex. ing the $\alpha=2$ cases for $\operatorname{Re}=10$, Fig. $11(\mathrm{~b})$, and for $\operatorname{Re}=40$, Fig. 12(b). In the first the phase lag is about $\pi / 2$, which is consistent with the similar local Sherwood numbers observed in Figs. 9(c) and 9(d), and in the second the phase lag is about $\pi / 4$.

A summary of the calculated maximum drag force, $F_{x \text {-max }}$, and the time-averaged Sherwood numbers, $\overline{\overline{\mathrm{Sh}}}$, is provided in Table IV. As shown in Table IV, $F_{x-\max }$ increases as Reynolds number increases. $F_{x-\max }$ also increases with increasing Womersley parameter and amplitude. For unsteady flow when $F_{x}=F_{x \text {-max }}$, the boundary layer thickness is thinner, which results in a larger viscous component of the drag force; also at this instant the inertial forces are larger, which creates a larger pressure difference across the cylinder and a larger pressure component of the drag force. $F_{x-\max }$ is an important consideration in the design of a system such as an artificial lung which requires low $F_{x-\max }$ but high $\overline{\overline{\mathrm{Sh}}}$. A low $F_{x \text {-max }}$ is important to decrease the strain experienced by the right side of the heart which pumps against the pressure drop for the device, and a high $\overline{\overline{\mathrm{Sh}}}$ is desired for maximum oxygen transport.

From Table IV, it is apparent that the Reynolds number has the largest impact on $\overline{\overline{\mathrm{Sh}}}$, which increases as Reynolds number increases. This is consistent with that observed for the steady-state case, $\alpha=0$ and $A=0$, which is also shown in the table. As described for steady flow using a boundary layer analysis, it is reasonable to expect $\overline{\overline{\mathrm{Sh}}}$ to be approximately proportional to $\mathrm{Re}^{0.5}$; this is observed. For $\alpha=0.25$, $\overline{\overline{\mathrm{Sh}}}$ decreases with increasing amplitude and all $\overline{\overline{\mathrm{Sh}}}$ are smaller than their steady-state counterparts. This can be explained from a quasi-steady-state point of view in which each instantaneous surface-averaged Sherwood number, $\overline{\mathrm{Sh}}$, is based on an instantaneous Reynolds number, $\operatorname{Re}_{I}=\operatorname{Re}[1$ $+A \sin (t)]$. Using this analysis, $\overline{\overline{\mathrm{Sh}}}$ decreases with increasing amplitude as observed for $\alpha=0.25$. For $\alpha=2$ and $\alpha=4, \overline{\overline{\mathrm{Sh}}}$ increases with increasing Womersley parameter and amplitude. The effect of pulsatility, as shown by varying the Womersley parameter and amplitude, on $\overline{\overline{\mathrm{Sh}}}$ is relatively small as compared to the effect of Reynolds number on $\overline{\overline{S h}}$. Within the range of variables considered, the maximum improvement due to pulsatility, as compared to the corresponding steady state value, varies between $14.4 \%$ and $20.9 \%$ for Re $=10$ to $40, \alpha=4$, and $A=0.75$.

The specific regime (no vortex, intermittent vortex, or persistent vortex) for each parameter set studied is also shown in Table IV. In general, by examining the $\overline{\overline{\text { Sh }}}$ data and associated specific regime in Table IV, it can be observed that better transport occurs for flow characterized by the intermittent region than flow characterized by the persistent region.

\section{CONCLUSIONS}

The influence of free-stream flow pulsation on the velocity and concentration fields around a cylinder is presented as a fundamental study for an artificial lung whose blood flow is driven by the heart. Design considerations require a high 
$\overline{\overline{\text { Sh }}}$ but low $F_{x-\max }$. For the artificial lung device which typically operates in the $1 \leqq \operatorname{Re} \lesssim 20$ and $\alpha \approx 0.25$ range with $A$ based on the cardiac output and compliance of the device, $A \approx 0.5-0.8$, no improvement due to pulsatility is observed when considering only a single cylinder, though better transport is achieved with higher Reynolds numbers. However, $F_{x \text {-max }}$, the drag force which the heart must pump against, also increases with Reynolds number. An acceptable maximum $F_{x-\max }$ would provide an upper limit on the selection of Re. In order to optimize the operating conditions and to predict the influence of flow pulsation on the transport of the device, the influence of other neighboring fibers, which are randomly placed in the fiber bundle, should be investigated for its effect on the flow and mass transport fields.

\section{ACKNOWLEDGMENTS}

The authors gratefully acknowledge financial support from the National Institute of Health under Grant No. NIH HL69420 and from the National Science Foundation through a graduate research fellowship awarded to J.R.Z.

${ }^{1}$ J. B. Zwischenberger, C. M. Anderson, K. E. Cook, S. D. Lick, L. F. Mockros, and R. H. Bartlett, "Development of an implantable artificial lung: Challenges and progress," ASAIO J. 47, 316 (2001).

${ }^{2}$ J. B. Zwischenberger and S. K. Alpard, "Artificial lungs: A new inspiration," Perfusion 17, 253 (2002).

${ }^{3}$ S. D. Lick and J. B. Zwischenberger, "Artificial lung: Bench toward bedside," ASAIO J. 50, 2 (2004).

${ }^{4}$ J. W. Haft, B. P. Griffith, R. B. Hirschl, and R. H. Bartlett, "Results of an artificial-lung survey to lung transplant program directors," J. Heart Lung Transplant 21, 467 (2002).

${ }^{5}$ R. Hilpert, "Warmeabgabe von geheizten Drahten und Rohren im Luftstrom," Forsch. Geb. Ingenieurwes. 4, 215 (1933).

${ }^{6} \mathrm{P}$. H. Vogtlander and C. A. P. Bakker, "An experimental study of mass transfer from a liquid flow to wires and gauzes," Chem. Eng. Sci. 18, 583 (1963).

${ }^{7}$ B. G. V. Zijnen, "Heat transfer from horizontal cylinders to a turbulent air flow,” Appl. Sci. Res., Sect. A 7, 205 (1958).

${ }^{8}$ V. V. Gnielinski, "Berechnung mittlerer warme- und stoffubergangskoeffizienten an laminar und turbulent uberstromten einzelkorpern mit hilfe einer einheitlichen gleichung," Forsch. Ingenieurwes. 41, 145 (1975).

${ }^{9}$ V. N. Kurdyumov and E. Fernandez, "Heat transfer from a circular cylinder at low Reynolds numbers," Trans. ASME, Ser. C: J. Heat Transfer 120, 72 (1998).

${ }^{10}$ E. M. Sparrow, J. P. Abraham, and J. C. K. Tong, "Archival correlations for average heat transfer coefficients for non-circular and circular cylinders and for spheres in cross-flow," Int. J. Heat Mass Transfer 47, 5285 (2004).

${ }^{11}$ C. T. Leung, N. W. M. Ko, and K. H. Ma, "Heat-transfer from a vibrating cylinder," J. Sound Vib. 75, 581 (1981).
${ }^{12}$ D. Karanth, G. W. Rankin, and K. Sridhar, "A finite-difference calculation of forced convective heat-transfer from an oscillating cylinder," Int. J. Heat Mass Transfer 37, 1619 (1994).

${ }^{13}$ J. Perwaiz and T. E. Base, "Heat-transfer from a cylinder and finned tube in a pulsating cross-flow," Exp. Therm. Fluid Sci. 5, 506 (1992).

${ }^{14}$ H. J. Sung, K. S. Hwang, and J. M. Hyun, "Experimental study on masstransfer from a circular-cylinder in pulsating flow," Int. J. Heat Mass Transfer 37, 2203 (1994).

${ }^{15} \mathrm{H}$. M. Badr, "Effect of free-stream fluctuations on laminar forced convection from a straight tube," Int. J. Heat Mass Transfer 40, 3653 (1997).

${ }^{16}$ G. E. Karniadakis, "Numerical simulation of forced-convection heattransfer from a cylinder in cross-flow," Int. J. Heat Mass Transfer 31, 107 (1988).

${ }^{17}$ R. J. Goldstein and J. Karni, "The effect of a wall boundary-layer on local mass-transfer from a cylinder in cross-flow," Trans. ASME, Ser. C: J. Heat Transfer 106, 260 (1984).

${ }^{18}$ S. Tiwari, G. Biswas, P. L. N. Prasad, and S. Basu, "Numerical prediction of flow and heat transfer in a rectangular channel with a built-in circular tube," Trans. ASME, Ser. C: J. Heat Transfer 125, 413 (2003).

${ }^{19}$ C. H. K. Williamson, "Sinusoidal flow relative to circular-cylinders," J. Fluid Mech. 155, 141 (1985).

${ }^{20} \mathrm{P}$. Justesen, "A numerical study of oscillating flow around a circularcylinder," J. Fluid Mech. 222, 157 (1991).

${ }^{21}$ H. M. Badr, S. C. R. Dennis, S. Kocabiyik, and P. Nguyen, "Viscous oscillatory flow about a circular-cylinder at small to moderate Strouhal number," J. Fluid Mech. 303, 215 (1995).

${ }^{22}$ C. F. Lange, F. Durst, and M. Breuer, "Momentum and heat transfer from cylinders in laminar crossflow at $10(-4) \Leftarrow \mathrm{Re} \Leftarrow 200$," Int. J. Heat Mass Transfer 41, 3409 (1998).

${ }^{23}$ S. V. Patankar, Numerical Heat Transfer and Fluid Flow (Hemisphere, New York, 1980).

${ }^{24}$ J. W. Demmel, S. C. Eisenstat, J. R. Gilbert, X. Y. S. Li, and J. W. H. Liu, "A supernodal approach to sparse partial pivoting," SIAM J. Matrix Anal. Appl. 20, 720 (1999).

${ }^{25} \mathrm{H}$. Watanabe and K. Iizuka, "The influence of dissolved-gases on the density of water," Metrologia 21, 19 (1985).

${ }^{26}$ G. S. Kell, "Effects of isotopic composition, temperature, pressure, and dissolved-gases on density of liquid water," J. Phys. Chem. Ref. Data 6, 1109 (1977).

${ }^{27}$ S. C. R. Dennis and G. Z. Chang, "Numerical solutions for steady flow past a circular cylinder at Reynolds numbers up to 100," J. Fluid Mech. 42, 471 (1970).

${ }^{28}$ A. E. Hamielec and J. D. Raal, "Numerical studies of viscous flow around circular cylinders," Phys. Fluids 12, 11 (1969).

${ }^{29}$ S. J. D. Dalessio and S. C. R. Dennis, "A method of domain decomposition for calculating the steady flow past a cylinder," J. Eng. Math. 28, 227 (1994).

${ }^{30}$ S. J. D. Dalessio and S. C. R. Dennis, "A vorticity model for viscous-flow past a cylinder," Comput. Fluids 23, 279 (1994).

${ }^{31}$ W. M. Deen, Analysis of Transport Phenomena (Oxford University Press, New York, 1998).

${ }^{32}$ S. Whitaker, Elementary Heat Transfer Analysis (Pergamon, New York, 1976).

${ }^{33}$ A. Zukauskas, "Heat Transfer from Tubes in Crossflow," in Advances in Heat Transfer, edited by J. P. Hartnett and T. F. Irvine (Academic, New York, 1987), Vol. 18, p. 87. 